\title{
Pseudo Approximation Algorithms with Applications to Optimal Motion Planning*
}

\author{
Tetsuo Asano, ${ }^{1}$ David Kirkpatrick, ${ }^{2}$ and Chee Yap ${ }^{3}$ \\ ${ }^{1}$ School of Information Science, JAIST, \\ Asahidai, Tatsunokuchi, Ishikana 923-12, Japan \\ t-asano@jaist.ac.jp \\ ${ }^{2}$ Department of Computer Science, University of British Columbia, \\ Vancouver, British Columbia, Canada V6T 1 Z4 \\ kirk@cs.ubc.ca \\ ${ }^{3}$ Courant Institute, New York University, \\ 251 Mercer Street, New York, NY 10012, USA \\ yap@cs.nyu.edu
}

Abstract. We introduce a technique for computing approximate solutions to optimization problems. If $X$ is the set of feasible solutions, the standard goal of approximation algorithms is to compute $x \in X$ that is an $\varepsilon$-approximate solution in the following sense:

$$
d(x) \leq(1+\varepsilon) d\left(x^{*}\right),
$$

where $x^{*} \in X$ is an optimal solution, $d: X \rightarrow \mathbb{R}_{\geq 0}$ is the optimization function to be minimized, and $\varepsilon>0$ is an input parameter. Our approach is first to devise algorithms that compute pseudo $\varepsilon$-approximate solutions satisfying the bound

$$
d(x) \leq d\left(x_{R}^{*}\right)+\varepsilon R,
$$

where $R>0$ is a new input parameter. Here $x_{R}^{*}$ denotes an optimal solution in the space $X_{R}$ of $R$-constrained feasible solutions. The parameter $R$ provides a stratification of $X$ in the sense that (1) $X_{R} \subseteq X_{R^{\prime}}$ for $R<R^{\prime}$ and (2) $X_{R}=X$ for $R$ sufficiently large.

We first describe a highly efficient scheme for converting a pseudo $\varepsilon$-approximation algorithm into a true $\varepsilon$-approximation algorithm. This scheme is useful because pseudo approximation algorithms seem to be easier to construct than $\varepsilon$-approximation algorithms. Another benefit is that our algorithm is automatically precision-sensitive.

\footnotetext{
* David Kirkpatrick was supported by the Natural Science and Engineering Research Council of Canada. Chee Yap was supported by NSF/ITR grant \#CCR-0082056.
} 
We apply our technique to two problems in robotics: (A) Euclidean Shortest Path (3ESP), namely the shortest path for a point robot amidst polyhedral obstacles in three dimensions, and (B) $d_{1}$-optimal motion for a rod moving amidst planar obstacles (1ORM). Previously, no polynomial time $\varepsilon$-approximation algorithm for (B) was known. For (A), our new solution is simpler than previous solutions and has an exponentially smaller complexity in terms of the input precision.

\section{Introduction}

The design of approximation algorithms is an important theme in the study of optimization problems. The standard goal here is to compute feasible solutions $x$ that are $\varepsilon$-approximate in the following sense. Suppose $X$ is the space of feasible solutions, and $d: X \rightarrow \mathbb{R}_{\geq 0}$ is the criterion for minimization. Then

$$
d(x) \leq(1+\varepsilon) d\left(x^{*}\right),
$$

where $x^{*}$ is an optimum solution.

Especially in geometric settings, we can often parametrize the space $X$ by a real parameter $R$ to yield subspaces $\left\{X_{R} \subseteq X: R \geq 0\right\}$ with two properties: (1) if $R<R^{\prime}$, then $X_{R} \subseteq X_{R^{\prime}}$, and (2) $X_{R}=X$ for $R$ sufficiently large. We call $R$ the search radius. Let $x_{R}^{*}$ denote the optimum solution in $X_{R}$. Then a pseudo $\varepsilon$-approximation algorithm is one ${ }^{1}$ that constructs $x$ satisfying

$$
d(x) \leq d\left(x_{R}^{*}\right)+\varepsilon R
$$

for any given $\varepsilon>0$ and $R \geq 0$. In many situations such algorithms are easier to construct than a true $\varepsilon$-approximation algorithm. (Intuitively, the parameter $R$ serves to offset the complexity attributable to the size of the search space by permitting a larger relative error.)

We will show that, under some fairly natural assumptions on $d\left(x_{R}^{*}\right)$, we can systematically convert a pseudo $\varepsilon$-approximation algorithm into an efficient $\varepsilon$-approximation algorithm which is automatically precision-sensitive. The advantage for this approach derives from (i) the relative ease of constructing pseudo approximation algorithms compared with approximation algorithms, (ii) the use of the above generic conversion scheme, and (iii) a clearer understanding of those aspects of the approximation process that are sensitive to the precision of the input (as well as the nature of this dependence).

Two NP-Hard Optimum Motion Planning Problems. Approximation algorithms take on a special significance when applied to problems that are provably intractable. We apply the above technique to derive approximation algorithms for two $N P$-hard problems in the area of robot motion planning. Although motion planning [1], [2], [8], [10], [23], [25] has been extensively studied since the early 1980s, very little is known about the problem of shortest length motion. Indeed, the only known efficient general algorithms apply

\footnotetext{
${ }^{1}$ In combinatorial optimization, the term "pseudo-approximation" sometimes refer to a feasible solution when the original constraints are relaxed.
} 
only to the case where robot body is a disk in two dimensions. This paucity of efficient algorithms is not for lack of interest in optimum motion (see below for an overview of the literature in the case of a rod). In retrospect, we now understand the lack of success:

(A) In three dimensions, Canny and Reif [6] show that the shortest path for a point robot moving amidst polyhedral obstacles is $N P$-hard to compute. This problem is known as Euclidean Shortest Path in three dimensions (3ESP).

(B) In two dimensions, Asano et al. [3] define the " $d_{1}$-distance" of a rod motion to be the length of the trajectory of the midpoint of the rod and prove the $d_{1}$-optimal motion of a rod amidst polygonal obstacles is $N P$-hard to compute. Let 1 ORM be the acronym for this problem. The rod is a directed line segment, and for now the reader may interpret the " $d_{1}$-distance" of a rod motion to be the length of the trajectory of the midpoint of the rod (see Appendix I for details).

Both (A) and (B) are the simplest optimum motion planning problems in dimensions 3 and 2, respectively, that go beyond a planar disk robot. These $N P$-hardness results were unexpected when they were first obtained. In any case, they immediately motivated the search for approximation algorithms. Papadimitriou [15] gave the first approximation algorithm for Problem (A). This was improved and sharpened by Choi et al. [7]. Sellen et al. [19] further constructed the first precision-sensitive algorithm that constructs the true combinatorially shortest path sequence, as well as an $\varepsilon$-approximate path on this sequence.

For Problem (B), no previous $\varepsilon$-approximation algorithm was known. Asano et al. [3] provided an approximation algorithm that, for any $\varepsilon, \varepsilon^{\prime}>0$, produces a motion $\mu$ for the rod satisfying

$$
d_{1}(\mu) \leq(1+\varepsilon) d_{1}\left(\mu^{*}\right)+O\left(n^{2} \varepsilon^{\prime}\right)
$$

where $\mu^{*}$ is a $d_{1}$-optimal motion. The algorithm runs in time is $O\left(n^{4} \alpha(n) K\right)$ where $\alpha(n)$ is the inverse Ackermann function and

$$
K=K\left(L, \varepsilon, \varepsilon^{\prime}\right)=\frac{L-\log \varepsilon^{\prime}}{\log \left(1+\varepsilon L^{-L}\right)} .
$$

It is assumed that the input description involves only rational numbers, $L$ is the maximum bit length of the input integers, and $n$ is the number of obstacle corners. We use the same parameters in the algorithms of this paper. All complexity bounds in this paper are in the algebraic complexity model [7].

Unfortunately, this algorithm falls short of being a true $\varepsilon$-approximation algorithm in two respects. First, the analysis assumes a quantitative form of their shortest path characterization that, while plausible, has not actually been proved. Second, the bound has an additive term " $+O\left(n^{2} \varepsilon^{\prime}\right)$ ", which is independent of the main term " $(1+\varepsilon) d_{1}\left(\mu^{*}\right)$ ". Since $\varepsilon^{\prime}$ can be set as small as we like (at some increase in the running time), we will obtain an $\varepsilon$-approximation algorithm if we could choose it so that the main term dominates the additive term. To do this, we need some a priori lower bound on $d_{1}\left(\mu^{*}\right)$ and then choose $\varepsilon^{\prime}$ accordingly.

Both of these deficiencies are addressed in this paper. A lower bound on $d_{1}\left(\mu^{*}\right)$ is established in Section 7, which overcomes the second of these shortcomings. With regard 
to the first, while we can prove a quantitative form of the shortest path characterization theorem, the associated path complexity bound turns out to be poorly suited for the purposes of constructing an efficient $\varepsilon$-approximation algorithm because of its dependence on $L$. (Recall that the characterization of shortest paths for a point or disk has no such dependence.) The development of an $\varepsilon$-approximation algorithm for 1ORM that depends only on a qualitative shortest path characterization theorem was the driving motivation for a pseudo approximation framework that forms the key innovation of this paper.

This general framework shows how to start with any pseudo approximation algorithm satisying some simple properties and derive an efficient $\varepsilon$-approximation algorithm. While the formal definition of "pseudo approximations" is somewhat specialized for the current applications, it should be understood that the intuitive concept of a pseudo approximation is that of having an arbitrary additive term which can be made as small as one likes. In this sense, almost any straightforward discretization of a continuous optimization problem is a pseudo approximation. Therein lies the power of this framework.

Optimum Motion Planning of a Rod. The configuration space for the motion of a rod is three-dimensional (as for 3ESP) but it is non-Euclidean. A rod is a fixed directed line segment $A B$ of unit length, as shown in Fig. 1(a). The problem is to find a motion of the rod from some initial placement $Z_{0}$ to some final placement $Z_{1}$ while confined to a closed polygonal region $\Omega \subseteq \mathbb{R}^{2}$. The complementary set $\mathbb{R}^{2} \backslash \Omega$ is the obstacle set. See Fig. 1(b). Let $F$ be a fixed point on $A B$, called the focus point. If $\mu$ is a motion of the rod, the corresponding trajectory of $F$ is called the trace of $\mu$; the length of this trace is the $d_{1}$-distance of $\mu$, denoted by $d_{1}(\mu)$. If $\mu^{*}$ has the minimum $d_{1}$-distance among

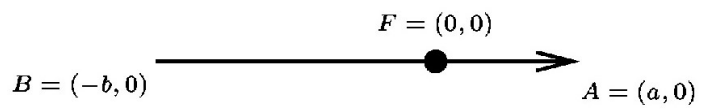

(a)

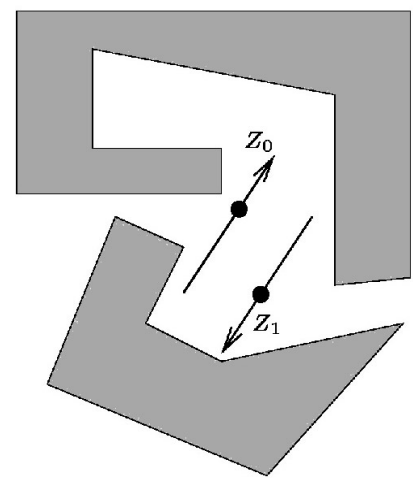

(b)

Fig. 1. (a) Canonical position of $\operatorname{rod} A B$ with focus $F$. (b) Moving from $Z_{0}$ to $Z_{1}$ amidst polygonal obstacles (shaded area). 
motions from $Z_{0}$ to $Z_{1}$, we write $d_{1}\left(Z_{0}, Z_{1}\right)=d_{1}\left(\mu^{*}\right)$ and call $\mu^{*}$ a $d_{1}$-optimum motion. Thus the input to our algorithms is $\left(Z_{0}, Z_{1}, \Omega\right)$, plus other input parameters such as $\varepsilon$ when appropriate. Refer to Appendix I for further background, including a description of the free space $F P=F P(\Omega)$. Although we focus on $d_{1}$-optimality, other notions of optimality have been considered in the interesting history of this problem:

- The oldest work here is Kakeya's problem [5], which asks for the smallest area swept by the rod while moving it from any position to its "dual position" (this is the position reached by rotating the rod $180^{\circ}$ about its midpoint), in the absence of obstacles. The obvious rotation motion sweeps out an area of $\pi / 4$ which turns out to be far from optimal.

- Ulam's problem [24], [14] is to minimize the average length of the trajectories of the two endpoints $A, B$ while moving from $Z_{0}$ to $Z_{1}$. This is also in the absence of obstacles. Icking et al. [12] introduced the Cauchy surface area formula as a tool for analyzing such motions. They define the $d_{n}$-distance for rod motion, where $n \geq 1$ is any integer or $n=\infty$. This distance is a metric for $n \geq 2$, and Ulam's problem corresponds to $d_{2}$.

- An optimality notion that is not based on distance is to maximize the minimum clearance (i.e., distance to the nearest obstacle) of the motion. Here, Ó’Dúnlaing et al. [13], [21], [22] gave a quadratic time solution based on the retraction approach.

- Although the $d_{1}$-distance is not a metric, it is a natural and interesting measure. Restricted forms of $d_{1}$-motions were investigated by Papadimitriou and Silverberg [16], Sharir [20] and O'Rourke [14]. The surprising result of Asano et al. [3] is that $d_{1}$-optimal motion is $N P$-hard whenever $F$ lies in the relative interior of the rod. The authors recently succeeded in extending the $N P$-hardness result to the case when $F$ is at an endpoint of the rod. This result will be presented in the near future.

\section{Contributions of This Work.}

(I) We introduce the framework of pseudo approximation algorithms and derive an efficient search scheme for converting any suitable pseudo approximation algorithm into a true $\varepsilon$-approximation algorithm. The approximation algorithm is precision sensitive.

(II) We construct an efficient $\varepsilon$-approximation algorithm for the $d_{1}$-optimal motion of a rod. This is based on a new and simplified strongly polynomial pseudo $\varepsilon$-approximation algorithm. Instead of (3), our pseudo approximate motion $\mu$ satisfies

$$
d_{1}(\mu) \leq d_{1}\left(\mu_{R}^{*}\right)+\varepsilon R,
$$

where $\varepsilon>0$ and $R>0$ are arbitrary parameters. Here $\mu_{R}^{*}$ is the optimal $d_{1}$ motion when the trace is restricted to a ball of radius $R$ centered at the initial position of the focus (denoted by $F\left[Z_{0}\right]$ ).

(III) As another application, we provide a new $\varepsilon$-approximation algorithm for the shortest path for a point amidst polyhedral obstacles in three-dimensions. This algorithm depends logarithmically on the input precision parameter $L$. Previous $\varepsilon$-approximation algorithms are polynomial (or even exponential) in $L$. Being 
simpler than previous solutions, it is possibly implementable (a hope that was expressed for the algorithm in [15]). See also [19] for some experimental results.

(IV) An improved analysis of the boundary $\partial F P$ of the space of free placements of a rod. This boundary is important for $d_{1}$-optimal motion. We introduce a 2-complex structure for this set.

Paper Overview. In Section 2 we introduce the framework of pseudo approximations. Section 3 shows how to convert a pseudo approximation algorithm into an $\varepsilon$ approximation algorithm. Section 4 gives a pseudo approximation algorithm for 3ESP, leading to a new $\varepsilon$-approximation algorithm. Section 5 reviews known results about the local structure of $d_{1}$-optimal rod motion. Based on this, we present in Section 6 a pseudo approximation algorithm for $d_{1}$-optimal rod motion (1ORM). Section 7 proves a lower bound on any non-zero $d_{1}$-distance. We conclude in Section 8. Appendices I and II summarize some background on $d_{1}$-optimal rod motion.

\section{The Pseudo Approximation Framework}

We describe an abstract framework for pseudo approximation algorithms.

Suppose that we are searching for an optimum solution $x^{*}$ in a search space $X$, where optimality is based on minimizing the function $d: X \rightarrow \mathbb{R}_{\geq 0}$. Assume that the space $X$ that has been "stratified" into the sets $\left\{X_{R} \subseteq X: R \geq 0\right\}$ such that (a) $X_{R} \subseteq X_{R^{\prime}}$ for $R<R^{\prime}$, and (b) we know a value $R^{*}$ such that $X=X_{R^{*}}$. The parameter $R$ should be interpreted as the "search radius".

For each $R \geq 0$, let $x_{R}^{*} \in X_{R}$ denote an optimum solution in $X_{R}$. It follows that

$$
d\left(x_{R}^{*}\right) \geq d\left(x_{R^{\prime}}^{*}\right) \geq d\left(x^{*}\right)
$$

for $R<R^{\prime}$. Now assume that we have a pseudo approximation function,

$$
\pi:(0,1] \times \mathbb{R}_{\geq 0} \rightarrow X
$$

such that for all $\varepsilon \in(0,1]$ and $R \geq 0, \pi(\varepsilon, R) \in X_{R}$ (and hence $\left.d(\pi(\varepsilon, R)) \geq d\left(x_{R}^{*}\right)\right)$ and

$$
d(\pi(\varepsilon, R)) \leq d\left(x_{R}^{*}\right)+\varepsilon R .
$$

Since the parameter $\varepsilon>0$ in (6) is fixed throughout this discussion, we may suppress it: we simple write " $\pi(R)$ " instead of " $\pi(\varepsilon, R)$ " where we view $\pi$ as the function $\pi: \mathbb{R}_{\geq 0} \rightarrow X$.

There is one additional property we need:

$$
d\left(x^{*}\right) \leq R \quad \Longrightarrow \quad d\left(x_{R}^{*}\right)=d\left(x^{*}\right) .
$$

This says that the search radius parameter $R$ has some direct correlation with $d\left(x_{R}^{*}\right)$ (i.e., the stratification of $X$ is tied to the underlying cost function). 
Some Claims. To interpret what the following claims say, it is convenient to call $R$ a high value in case $d(\pi(R)) \leq R$, and a low value otherwise.

Claim 1. If $R$ is a high value, i.e., $d(\pi(R)) \leq R$, then $R \geq d\left(x^{*}\right)$ and $d\left(x^{*}\right)=d\left(x_{R}^{*}\right)$.

Proof. The first relation follows from $R \geq d(\pi(R)) \geq d\left(x_{R}^{*}\right) \geq d\left(x^{*}\right)$. The second relation $d\left(x_{R}^{*}\right)=d\left(x^{*}\right)$ is then a consequence of the "correlation property" (7).

Claim 2. If $R$ is a low value, i.e., $d(\pi(R))>R$, then $d\left(x^{*}\right)>R(1-\varepsilon)$.

Proof. By way of contradiction, suppose $d\left(x^{*}\right) \leq R(1-\varepsilon)$. Then, by (7), $d\left(x_{R}^{*}\right)=$ $d\left(x^{*}\right)$. Hence $d(\pi(R)) \leq d\left(x_{R}^{*}\right)+\varepsilon R=d\left(x^{*}\right)+\varepsilon R \leq R(1-\varepsilon)+\varepsilon R=R$. This gives the desired contradiction.

Claim 3. Fix any constant $\alpha>1$. If $R_{\min }$ is a low value, $R_{\max }$ is a high value and $R_{\max } \leq \alpha R_{\min }$, then $d\left(\pi\left(R_{\max }\right)\right)<d\left(x^{*}\right)(1+\alpha(\varepsilon /(1-\varepsilon)))$.

Proof. Since $R_{\min }$ is a low value it follows from Claim 2 that

$$
d\left(x^{*}\right)>(1-\varepsilon) R_{\min } .
$$

Thus

$$
\begin{aligned}
& d\left(\pi\left(R_{\max }\right)\right) \leq d\left(x_{R_{\max }}^{*}\right)+\varepsilon R_{\max } \quad \text { (equation (6)) } \\
& =d\left(x^{*}\right)+\varepsilon R_{\max } \quad(\text { Claim } 1) \\
& \leq d\left(x^{*}\right)+\varepsilon \alpha R_{\min } \\
& <d\left(x^{*}\right)+\varepsilon \frac{\alpha d\left(x^{*}\right)}{1-\varepsilon} \quad \text { (equation (8)) } \\
& =d\left(x^{*}\right)\left(1+\frac{\alpha \varepsilon}{1-\varepsilon}\right) \text {. }
\end{aligned}
$$

Corollary 1. Assuming $\varepsilon \leq \frac{1}{2}$, if $R_{\min }$ is a low value, $R_{\max }$ is a high value and $R_{\max } \leq$ $2 R_{\text {min }}$, then

$$
d\left(\pi\left(R_{\max }\right)\right)<d\left(x^{*}\right)(1+3 \varepsilon) .
$$

We will also need the following:

Claim 4. If $R$ is a low value and $d\left(x_{R}^{*}\right)=d\left(x^{*}\right)$, then $d(\pi(R))<d\left(x^{*}\right) /(1-\varepsilon)$.

Proof. By Claim 2, $d(\pi(R))>R$ implies $d\left(x^{*}\right)>R(1-\varepsilon)$. Thus $d(\pi(R)) \leq$ $d\left(x_{R}^{*}\right)+\varepsilon R=d\left(x^{*}\right)+\varepsilon R<d\left(x^{*}\right)+\varepsilon d\left(x^{*}\right) /(1-\varepsilon)=d\left(x^{*}\right) /(1-\varepsilon)$. 
Suppose our pseudo approximation function $\pi(R)$ has the following monotonicity property:

$$
d(\pi(R-r)) \geq d(\pi(R)) \quad \text { for all } \quad r \geq 0 .
$$

This property is not hard to ensure in the two main examples in this paper. It has an interesting consequence:

Claim 5. If the pseudo approximation function satisfies (10), then it induces a 0-1 ordering of the real numbers in the following sense: if $R$ is a low value, then $R-r$ is a low value, for all $r \geq 0$.

Proof. If $R$ is a low value, then $d(\pi(R))>R$. Hence $d(\pi(R-r)) \geq d(\pi(R))>R>$ $R-r$, i.e., $R-r$ is a low value.

\section{Conversion to Precision-Sensitive $\varepsilon$-Approximations}

We now show how any pseudo approximation algorithm in the previous section can be converted into an $\varepsilon$-approximation algorithm.

Our analysis uses two simple assumptions: $(1) d(\pi(0))>0$ (i.e., 0 is a low value) and (2) $\varepsilon \leq \frac{1}{2}$. Note that if (1) fails, then $d(\pi(0))=0$ and $\pi(0)$ is already an optimal solution. As for (2), we could have used any $C<1$ in place of $\frac{1}{2}$.

We first present a simple binary search method. The idea is to exploit the corollary to Claim 3, by maintaining a pair $\left(R_{\min }, R_{\max }\right)$ satisfying the invariant that $R_{\min }$ is a low value and $R_{\max }$ is a high value. We start from the largest search radius $R^{*}$. At each iteration, we halve the gap $R_{\max }-R_{\min }$, halting when the gap is at most $R_{\min }$. The basic comparison of the binary search is testing if a number $R>0$ is high or low. This amounts to a call of the pseudo approximation function to compute $d(\pi(R))$ and comparing it with $R$.

\section{SIMPLE BINARY SEARCH}

OUTPUT: $R$ such that $d(\pi(R)) \leq d\left(x^{*}\right)(1+3 \varepsilon)$.

BASE CASE: if $R^{*}$ is low, Return $R^{*}$.

$R_{\min } \leftarrow 0$ and $R_{\max } \leftarrow R^{*}$.

Do \{

$R \leftarrow\left(R_{\max }+R_{\min }\right) / 2$.

If $R$ is low, then $R_{\text {min }} \leftarrow R$

else $R_{\text {max }} \leftarrow R$.

\} while $\left(R_{\max }>2 R_{\min }\right)$

Return $R_{\max }$.

Correctness: The base case is justified by Claim 4 (use the fact $1 /(1-\varepsilon)=1+\varepsilon+$ $\left.\varepsilon^{2} /(1-\varepsilon) \leq 1+2 \varepsilon\right)$. The loop invariant is maintained as we update $R_{\min }$ and $R_{\max }$. Upon termination, the output is correct by the corollary of Claim 3. 
Complexity: We claim that the number of iterations is at most $2+\lg \left(R^{*} / d\left(x^{*}\right)\right)$. Each iteration reduces the gap $R_{\max }-R_{\min }$ by a factor of two, and the initial gap is $R^{*}$. So it suffices to show that the final gap is at least $d\left(x^{*}\right) / 4$. Let $r_{\max }$ and $r_{\min }$ be the values of the variables $R_{\max }$ and $R_{\min }$ in the previous iteration (i.e., the last iteration for which $\left.R_{\max }>2 R_{\min }\right)$. Now, $\left(r_{\max }+r_{\min }\right) / 2$ is equal to either $R_{\max }$ or $R_{\min }$. Hence, either $R_{\max }=\left(r_{\max }+r_{\min }\right) / 2$ and $R_{\min }=r_{\min }$ or $R_{\max }=r_{\max }$ and $R_{\min }=\left(r_{\max }+r_{\min }\right) / 2$. Therefore, since $r_{\max }>2 r_{\min }$,

$$
R_{\max }-R_{\min }=\left(r_{\max }-r_{\min }\right) / 2>r_{\max } / 4 \geq R_{\max } / 4 \geq d\left(x^{*}\right) / 4
$$

(the last inequality follows from Claim 1). This concludes the proof.

Geometric Search. We can significantly speed up the above search using the following two-tiered search:

\section{GEOMETRIC SEARCH}

OUTPUT: $R$ such that $d(\pi(R)) \leq d\left(x^{*}\right)(1+3 \varepsilon)$.

0. BASE CASE: if ( $R^{*}$ is low) then Return $R^{*}$.

1. KEY TEST: If ( $R=1$ is low)

then $R_{\min }=1, k=\left\lceil\lg \lg R^{*}\right\rceil$, and go to Search 2 directly. else $R_{\min }=\frac{1}{2}, k=0$, and go to Search 1 first.

2. SEARCH 1: // Now $R_{\min } 2^{2^{k}}$ is high

While $\left(R_{\min }\right.$ is high) do

$$
R_{\min } \leftarrow R_{\min } 2^{-2^{k}} ; k \leftarrow k+1 ;
$$

// Invariant 1: $R_{\min } 2^{2^{k}}$ is high

3. SEARCH 2: // Now $R_{\min }$ is low and $R_{\min } 2^{2^{k}}$ is high

$l o \leftarrow 0 ; h i \leftarrow 2^{k}$;

While $(h i-l o>1)$ do

// Invariant 2: $R_{\min } 2^{l o}$ is low, $R_{\min } 2^{h i}$ is high

$m \leftarrow(h i+l o) / 2$;

if $\left(R_{\min } 2^{m}\right.$ is low) then $l o \leftarrow m$;

else $h i \leftarrow m$;

$R_{\min } \leftarrow R_{\min } 2^{l o} ; R_{\max } \leftarrow R_{\min } 2^{h i} ;$

4. Return $R_{\max }$.

Correctness: The base case is justified as for the simple binary search. The two loop invariants are easily verified. The final return statement is again justified by the corollary to Claim 3.

Complexity: After $k \geq 1$ iterations in Search 1, the variable $R_{\min }$ reaches the value $r_{k}:=2^{-2^{0}} 2^{-2^{0}} 2^{-2^{1}} \cdots 2^{-2^{k-1}}=2^{-2^{k}}$. If $r_{k}$ is high, then $r_{k} \geq d\left(x^{*}\right)$; hence $2^{2^{k}} \leq 1 / d\left(x^{*}\right)$ and so $k \leq \lg \lg \left(1 / d\left(x^{*}\right)\right)$. However, the total number of iterations in Search 1 is just one more than the last value of $k$ for which $r_{k}$ is high. This proves the first search has $\leq 1+\lg \lg \left(1 / d\left(x^{*}\right)\right)$ iterations. In Search 2 we begin with a pair of values $(l o, h i)=$ 
$\left(0,2^{k}\right)$. If we came directly from Step 1 (Key Test), then $k=\left\lceil\lg \lg R^{*}\right\rceil$; otherwise we just completed Search 1 and $k \leq\left\lfloor\lg \lg \left(1 / d\left(x^{*}\right)\right)\right\rfloor$. We conclude that

$$
k \leq \max \left\{\left\lceil\lg \lg R^{*}\right\rceil,\left\lfloor\lg \lg \left(1 / d\left(x^{*}\right)\right)\right\rfloor\right\} .
$$

Initially $h i-l o=2^{k}$ but finally we have $\frac{1}{2}<h i-l o \leq 1$. The number of iterations is $\leq 1+k=1+\max \left\{\left\lceil\lg \lg R^{*}\right\rceil,\left\lfloor\lg \lg \left(1 / d\left(x^{*}\right)\right)\right\rfloor\right\}$.

In summary:

Lemma 2. The geometric search procedure determines $R$ such that $d(\pi(R)) \leq d\left(x^{*}\right)$ $(1+3 \varepsilon)$ using at most

$$
2+\max \left\{\left\lceil\lg \lg R^{*}\right\rceil, 2\left\lfloor\lg \lg \left(1 / d\left(x^{*}\right)\right)\right\rfloor\right\}
$$

calls to the pseudo approximation function.

Let us simply write $\max \left\{\left\lceil\lg \lg R^{*}\right\rceil, 2\left\lfloor\lg \lg \left(1 / d\left(x^{*}\right)\right)\right\rfloor\right\}=\Theta\left(\lg \lg \left(R^{*} / d\left(x^{*}\right)\right)\right)$. As a corollary, if computing $\pi(R)$ and $d(\pi(R))$ takes time $T=T\left(\varepsilon, R^{*}\right)$, we achieve an $\varepsilon$-approximation algorithm whose time is $O\left(T \times \lg \lg \left(R^{*} / d\left(x^{*}\right)\right)\right)$.

Note that Search 2 can be viewed as a "geometric mean" search: we can view the search as maintaining an interval $\left(R_{\min }, R_{\max }\right)$ in which each "halving step" involves replacing one endpoint of the interval by the geometric mean $\sqrt{R_{\min } R_{\max }}$. Even Search 1 can be interpreted in this way (except that in the absence of a low value, we replace $R_{\min }$ by 1$)$. This explains why we call this search method "Geometric Search".

Note that when $R$ is high, the combination of Search 1 and Search 2 is really a disguised form of "unbounded search" in the sense of Bentley and Yao [4]. The difference is that standard unbounded search uses an absolute error bound, while we use a relative error bound (see corollary to Claim 3 ). Our complexity bound is $2+\left\lfloor\lg \lg \left(1 / d\left(x^{*}\right)\right)\right\rfloor$. Known techniques for unbounded searching can be applied here to improve the upper bound to $\left\lfloor\lg \lg \left(1 / d\left(x^{*}\right)\right)\right\rfloor+o\left(\lg \lg \left(1 / d\left(x^{*}\right)\right)\right)$. We leave such improvements as an exercise.

One variant is to compute an a priori lower bound $r^{*}$ on $d\left(x^{*}\right)$. We can perform a simultaneous search for the critical value radius from above (starting from $R^{*}$, as in Search 1) and from below (starting from $r^{*}$ ). The number of iterations is then

$$
\min \left\{\lg \lg \left(R^{*} / d\left(x^{*}\right)\right), \lg \lg \left(d\left(x^{*}\right) / r^{*}\right)\right\} .
$$

In this way, we lose precision sensitivity but gain the potential to have speed up when $d\left(x^{*}\right)$ is very large (near $\left.R^{*}\right)$ or very small (near $r^{*}$ ).

Precision-Sensitive Solution. The running time of our $\varepsilon$-approximation algorithm depends on $d\left(x^{*}\right)$. In our applications below, $R^{*} \leq c^{L}$ for some $c>1$ and the maximum bit length $L$ of the input numbers. Hence if $\bar{d}\left(x^{*}\right)<c^{-L}$, then $\lg \lg \left(R^{*} / d\left(x^{*}\right)\right)=$ $\Theta\left(\lg \lg \left(1 / d\left(x^{*}\right)\right)\right)$; otherwise $\lg \lg \left(R^{*} / d\left(x^{*}\right)\right)=\Theta\left(\lg \lg \left(R^{*}\right)\right)$. We call $\lg \left(1 / d\left(x^{*}\right)\right)$ the "output precision" of the problem instance since it is proportional to the number of bits needed to express $d\left(x^{*}\right)$ to within a constant factor. In this sense we say that our algorithm is precision sensitive. Note that despite being sensitive to $d\left(x^{*}\right)$, our algorithm does not explicitly know $x^{*}$ or $d\left(x^{*}\right)$. 
The notion of precision sensitivity was first introduced in [7]. It should be noted that the 3ESP approximation algorithm there has a stronger objective than our version here: their objective was to determine a sequence of edges that determines the shortest path, as well as to determine an $\varepsilon$-approximate feasible motion along this sequence. Call this the "Combinatorial 3ESP Approximation Problem". In this version it is natural to define the "output precision" as $\log (1 / \Delta)$ where $\Delta=d\left(x^{*}\right)-d\left(x_{2}\right)$ and $x_{2}$ is a combinatorially distinct next shortest path. The gap $\Delta$ is a measure of the necessary number of bits that must be evaluated if we want to distinguish $x^{*}$ from $x_{2}$. The current best lower bound for non-zero $\Delta$ is doubly exponentially small ( $\operatorname{so} \lg (1 / \Delta)$ is single exponential). The precision-sensitive algorithm of [7] is only polynomial in the output precision, and hence the overall algorithm is not known to be polynomial time. In contrast, for our two applications below, we show a linear upper bound on $\lg \left(1 / d\left(x^{*}\right)\right)$ and so the running time of our algorithms are actually logarithmic in the output precision.

\section{Approximation Algorithm for 3ESP}

We now construct a pseudo approximation algorithm for 3ESP that has the properties required for our binary search method in the previous section. This serves three purposes: (1) It gives the first indication that our abstract setting in the previous section is nonvacuous. (2) It will serve as a model for our next section, where we give a pseudo approximation algorithm for rod motion. Additional complications will arise in the case of rod motion. (3) Finally, our new algorithm has advantages over previous solutions (some of which address shortest path problems in more general settings) [15], [8], [7], [1], [2], [19], [23]. One is its simplicity, thus making it a more likely candidate for implementation. Another is its running time complexity being $O(\log L)$, in contrast to previous algorithms whose complexity is polynomial (or even exponential) in $L$.

The main result of this section is this:

Theorem 3. There is a pseudo approximation algorithm for $3 E S P$ whose running time is $O\left(n^{4} / \varepsilon^{2}\right)$, or more precisely $O\left(n^{4} \varepsilon^{-2} \lg \lg \left(2^{L} / d\left(x^{*}\right)\right)\right)$.

This is a worst case complexity bound: the actual complexity is $O\left(\min \left\{n^{2} / \varepsilon,|A| \log \right.\right.$ $\left.\left.\left(n^{2} / \varepsilon\right)\right\}\right)$, where $|A|$ denotes the number of edges in the graph that is searched to find the shortest path. It is also worth noting that this complexity does not depend on the parameter $R$ (at least in the algebraic complexity model). While it is possible to convert this bound into the bit complexity model, a tight analysis can be intricate. Reference [7] is one of the few papers that gave a careful accounting of the bit complexity of an approximation algorithm.

Coupled with the geometric search procedure in the previous section, and using the estimate $R^{*}=2^{L}$, we obtain an $\varepsilon$-approximation algorithm with complexity

$$
O\left(n^{4} \varepsilon^{-2} \lg \lg \left(2^{L} / d\left(x^{*}\right)\right)\right) .
$$

As this expression depends on $d\left(x^{*}\right)$, to obtain an a priori bound on the running time, we need a lower bound on $d\left(x^{*}\right)$ in terms of the input parameters. This is not hard: we may 
assume $Z_{0} \neq Z_{1}$ or else the problem is trivial. Then we obtain $d\left(x^{*}\right) \geq\left\|Z_{0}-Z_{1}\right\| \geq c^{-L}$, for some constant $c>1$.

Input Parameters and Representation. Let $R \geq 1$ and $\varepsilon^{\prime}>0$ be given. (Later we will choose $\varepsilon^{\prime}=\varepsilon / 6$ where $\varepsilon$ is the corresponding pseudo approximation parameter.) There is also the the standard input $\left(Z_{0}, Z_{1}, \Omega\right)$ for motion planning, where $Z_{0}, Z_{1} \in \mathbb{R}^{3}$ and $\Omega \subseteq \mathbb{R}^{3}$ is a closed polyhedral set. There are $n$ obstacle edges and vertices, and the numbers used in their description are $L$-bit rational numbers, i.e., the numerators and denominators are $L$-bit integers. We write $\Omega_{R}$ for the restriction of $\Omega$ to a ball of radius $R$ centered at $Z_{0}$. Let $\mu_{R}^{*}$ denote a shortest path from $Z_{0}$ to $Z_{1}$ when restricted to $\Omega_{R}$. Our goal is to compute a path in $\Omega_{R}$ that is a "pseudo approximation" to $\mu_{R}^{*}$.

Fragment Visibility Graph. We describe a fragmentation of the obstacle edges (that depends on the parameters $R, \varepsilon^{\prime}$ and $\left.n\right)$ and a "visibilty graph" $F V G=(N, A ; W)$ on the resulting set of fragments. Specifically, $F V G$ is a weighted undirected graph, $N$ is the node set, $A$ is the arc set, and $W: A \rightarrow \mathbb{R}_{>0}$ is the weight function. Note that we use the node/arc terminology to avoid conflict with the vertex/edge terminology reserved for the obstacle set $\Omega$. First, we specify the node set $N$. For each obstacle edge $e \in \Omega$, we replace it with $e_{R}=e \cap \Omega_{R}$. Then we subdivide $e_{R}$ into $\left\lceil n / \varepsilon^{\prime}\right\rceil$ fragments, each of length at most $R \varepsilon^{\prime} / n$. Summed over all edges, we have $O\left(n^{2} / \varepsilon^{\prime}\right)$ fragments. Next, the node set $N$ comprises these fragments, including $Z_{0}$ and $Z_{1}$ as special fragments. The arc set $A$ comprises those pairs $\left(\sigma_{a}, \sigma_{b}\right)$ of fragments that are "weakly visible", i.e., there is a line segment $\left[\sigma_{a}^{*}, \sigma_{b}^{*}\right] \subseteq \Omega_{R}$ such that $\sigma_{a}^{*} \in \sigma_{a}$ and $\sigma_{b}^{*} \in \sigma_{b}$. We define the arc's weight $W\left(\sigma_{a}, \sigma_{b}\right)$, not as $\left\|\sigma_{a}^{*}-\sigma_{b}^{*}\right\|$, but as $\left\|\check{\sigma}_{a}-\check{\sigma}_{b}\right\|$ where $\check{\sigma}_{a}$ and $\check{\sigma}_{b}$ are the midpoints of $\sigma_{a}$ and $\sigma_{b}$. Refer to [7] for the details of these computations (deciding weak visibility, etc).

Given the fragment visibility graph $F V G=(N, A ; W)$, we can compute the shortest path $P^{*}$ from the source $Z_{0}$ to $Z_{1}$ using standard techniques such as Dijkstra's algorithm. Since $|A|=O\left(n^{4} / \varepsilon^{\prime 2}\right)$ this algorithm can be implemented in time $O(|N| \log |N|+|A|)=$ $O\left(n^{4} / \varepsilon^{\prime 2}\right)$, using Fibonacci heaps (e.g., [9]).

Relation between the Fragment Visibility Graph Shortest Path $P^{*}$ and the Restricted Euclidean Shortest Path $\mu_{R}^{*}$. The relation is indirect: from $P^{*}$, we construct a Euclidean motion $\mu\left(P^{*}\right)$. Then we derive a relation between $\mu\left(P^{*}\right)$ and the restricted Euclidean shortest path $\mu_{R}^{*}$. First we set out some basic properties that follow immediately from the definition of the fragmentation process:

Observation A. If $P$ is any single edge path in $F V G$ joining two fragments $\sigma_{a}$ and $\sigma_{b}$ of different obstacle edges, then their exists a Euclidean path $\mu(P)$ in $\Omega_{R}$ joining $\check{\sigma}_{a}$ and $\check{\sigma}_{b}$ such that $d(\mu(P)) \leq W(P)+\varepsilon^{\prime} R / n$.

Observation B. If $P$ is any path in $F V G$ joining two fragments $\sigma_{a}$ and $\sigma_{b}$ of the same obstacle edge, then their exists a Euclidean path $\mu(P)$ in $\Omega_{R}$ joining $\check{\sigma}_{a}$ and $\check{\sigma}_{b}$ such that $d(\mu(P)) \leq W(P)+\varepsilon^{\prime} R / n$. 
Observation C. If a Euclidean line segment $\mu$ in $\Omega_{R}$ joins two points $v_{a}$ and $v_{b}$, belonging to fragments $\sigma_{a}$ and $\sigma_{b}$ of different obstacle edges, then there exists a path $P(\mu)$ in $F V G$ joining fragments $\sigma_{a}$ and $\sigma_{b}$ such that $W(P(\mu)) \leq d(\mu)+\varepsilon^{\prime} R / n$.

Observation D. If a Euclidean path $\mu$ in $\Omega_{R}$ joins two points $v_{a}$ and $v_{b}$, belonging to fragments $\sigma_{a}$ and $\sigma_{b}$ of the same obstacle edge, then there exists a path $P(\mu)$ in $F V G$ joining fragments $\sigma_{a}$ and $\sigma_{b}$ such that $W(P(\mu)) \leq d(\mu)+\varepsilon^{\prime} R / n$.

Let $P$ be any path in the $F V G$ from $Z_{0}$ to $Z_{1}$. We can decompose $P$ into $k \leq$ $2 n$ subpaths $P_{1}, P_{2}, \ldots, P_{k}$, such that odd-indexed subpaths consist of single edges joining fragments of different obstacle edges, and even-indexed subpaths (possibly of zero length) join fragments of the same obstacle edge. Define $\mu(P)$ to be the Euclidean path formed by concatenation of $\mu\left(P_{1}\right), \ldots, \mu\left(P_{k}\right)$. By Observations A and $\mathrm{B}$, we have

$$
\begin{aligned}
d(\mu(P) & =\sum_{i=1}^{k} d\left(\mu\left(P_{i}\right)\right) \\
& \leq \sum_{i=1}^{k}\left[W\left(P_{i}\right)+\varepsilon^{\prime} R / n\right] \\
& \leq W(P)+2 \varepsilon^{\prime} R .
\end{aligned}
$$

On the other hand, if $\mu$ is any Euclidean path in $\Omega_{R}$, then $\mu$ can be decomposed into $k \leq 2 n$ subpaths $\mu_{1}, \mu_{2}, \ldots, \mu_{k}$ such that odd-indexed subpaths consist of single line segments joining fragments of different obstacle edges, and even-indexed subpaths (possibly of zero length) join points on fragments of the same obstacle edge. Define $P(\mu)$ to be the path in $F V G$ formed by concatenation of the paths $P\left(\mu_{1}\right), \ldots, P\left(\mu_{k}\right)$. By Observations $\mathrm{C}$ and $\mathrm{D}$, we have

$$
\begin{aligned}
W(P(\mu)) & =\sum_{i=1}^{k} W\left(P\left(\mu_{i}\right)\right) \\
& \leq \sum_{i=1}^{k}\left[d\left(\mu_{i}\right)+\varepsilon^{\prime} R / n\right] \\
& \leq d(\mu)+2 \varepsilon^{\prime} R .
\end{aligned}
$$

We now put these two steps together. Begin with the shortest path $P^{*}$ joining $Z_{0}$ and $Z_{1}$ in $F V G$. Using the construction above, we obtain a Euclidean path $\mu\left(P^{*}\right)$.

\section{Lemma 4.}

$$
d\left(\mu\left(P^{*}\right)\right) \leq d\left(\mu_{R}^{*}\right)+4 \varepsilon^{\prime} R
$$

Proof.

$$
\begin{aligned}
d\left(\mu\left(P^{*}\right)\right) & \leq W\left(P^{*}\right)+2 \varepsilon^{\prime} R & & (\text { by }(11)) \\
& \leq W\left(P\left(\mu_{R}^{*}\right)\right)+2 \varepsilon^{\prime} R & & \left(\text { by definition of } P^{*}\right) \\
& \leq d\left(\mu_{R}^{*}\right)+4 \varepsilon^{\prime} R & & (\text { by }(12)) .
\end{aligned}
$$


We make the final connection to the abstract binary search of the previous section. The restricted search space $X_{R}$ comprise all the Euclidean paths in $\Omega_{R}$. The pseudo approximation function $\pi:(0,1] \times \mathbb{R} \rightarrow X$ is given by $\pi(\varepsilon, R)=\mu\left(P^{*}\right)$ where $P^{*}$ is the shortest path in the Fragment Visibility Graph constructed with the parameters $\varepsilon^{\prime}=\varepsilon / 4$ and $R$. By Lemma 4, we know that $\pi$ is a pseudo approximation function. We need to verify the properties $d\left(\mu^{*}\right) \leq R$ implies $d\left(\mu_{R}^{*}\right)=d\left(\mu^{*}\right)$ (equation 7) and $R^{*} \leq 2^{L}$. However, they are easily seen. This proves the main result stated above.

Remark. This approach immediately generalizes to the shortest path for a point robot moving amidst polyhedral obstacles in any fixed dimension.

\section{Local Structure of Optimum Rod Motion}

In this section we give a top level overview of the underlying geometry of the optimal $d_{1}$-motion of a rod. This is a necessary prerequisite for the approximation algorithm to be presented in the next section. We defer details to the appendices because of some overlap with [3]. However, the current paper offers technical improvements and alternative treatment.

Decomposition of $\partial F P$. There are three known approaches to analyzing the structure of the free space $F P=F P(\Omega)$ of a rod. In [17] the set $F P$ is decomposed into cells using the notion of concept of clockwise and counterclockwise stops. The projection of these cells onto $\mathbb{R}^{2}$ gives a simple decomposition of $\Omega$ into planar regions. The second approach [21], [22] is based on the concept of Voronoi cells. Neither of these approaches are suitable for analyzing optimum $d_{1}$-motion. The third approach [3] was introduced for $d_{1}$-optimal motion, and is based on a cell decomposition of $\partial F P$. This boundary can be decomposed into a 2-complex comprising 2-cells (called patches), 1-cells (called edges) and 0 -cells (called vertices). The details are given Appendix II. In particular, it is shown (Theorem 8 ) that the number of patches, edges and vertices in the complex is $O\left(n^{2}\right), O\left(n^{3}\right)$ and $O\left(n^{3}\right)$, respectively.

Similar to retraction-based motion planning [13], our goal is to construct a onedimensional complex (i.e., a skeleton) in which optimum motion can be found. The obvious place to look is to consider the subcomplex $K$ of $\partial F P$ comprising the edges and vertices. The skeleton we seek will clearly need to augment $K$ with additional edges to ensure global connectivity. However, it turns out that no finite number of edges suffices for $d_{1}$-optimum motion.

On Stopover Curves and Mirrors. One can still hope for some "parametrized form" of the skeleton that can reduce optimal motion to a finite graph search. To see how this might work, consider the well-understood problem of optimum motion $\mu^{*}$ for the unit disk in the plane [11]. Here, the circular arcs of radius $\frac{1}{2}$ and centered at convex corners are called displaced corners [3]. They are important because the trace of $\mu^{*}$ is non-straight only by incorporating parts of these arcs. The optimal trace will join and leave such circular arcs at a tangent. If every tangent is potentially part of an optimum 
motion, there is no finite graph that we can construct. However, it turns out that we can construct a finite search graph in which these displaced corners are used as "parametrized vertices". The analog of these displaced corners for optimal $d_{1}$-optimal rod motion is called stopover curves [3]. Each stopover curve is simply the trace of a corresponding stopover edge of $\partial F P$. Each placement $Z$ in a stopover curve is constricted in the sense that rotation about the focus $F$ is impossible: the rod is stopped by two obstacle features, one acting as a clockwise stop, the other as a counterclockwise stop. There are three kinds of stopover curves: (1) a circular edge defined by a concave corner $C$, (2) an elliptic edge defined by a pair to distinct walls $W_{1}, W_{2}$, and (3) a conchoidal edge defined by a convex corner $C$ and a wall $W$. The traces of these edges are parts of a circle, an ellipse or an conchoid. In addition to these cases, there is a fourth kind of constricted edge: this is defined by two corners and the trace is a straight line segment. We do not consider this a stopover curve. See Appendix II for more details.

The number of stopover edges is $O\left(n^{3}\right)$ and we might hope for a finite search graph using these curves as "parametrized vertices" in the search graph. However, this hope is dashed by the phenomenon of mirrors. In a $d_{1}$-optimal motion, the traces can "reflect" off the mirrors following the law of reflection (see Appendix II). In fact, mirrors are the reason for $N P$-hardness [3]. Fortunately, when we seek approximate motions, we can avoid mirrors altogether.

Locally $d_{1}$-Optimal Motion. In order to make further progress, we need to understand what can happen "locally" in a $d_{1}$-optimal motion $\mu:[0,1] \rightarrow F P$. Intuitively, the trace $F \mu:[0,1] \rightarrow \mathbb{R}^{2}$ must travel along a straight line unless it is forced by some obstacle features to turn or bend.

Let $X$ be a metric space with metric $d(x, y)$, and fix a continuous function (curve) $f:[0,1] \rightarrow X$. If $\varepsilon>0$ and $0<t_{0}<1$, we call an open interval $I$ an $(\varepsilon, f)$ neighborhood of $t_{0}$ if $t_{0} \in I$ and for all $t \in I, d\left(f(t), f\left(t_{0}\right)\right)<\varepsilon$. We may also call $I$ an $f$-neighborhood of $t_{0}$ if it is an $(\varepsilon, f)$-neighborhood for some $\varepsilon>0$. If $P$ is any property of curves, we say that $f$ satisfies $P$ at $t_{0}\left(0<t_{0}<1\right)$ if there exists $\varepsilon=\varepsilon\left(t_{0}\right)>0$ and an $(\varepsilon, f)$-neighborhood $I$ of $t_{0}$ such that the restriction $f \mid I$ of $f$ to $I$ satisfies property $P$. If $f$ satisfies $P$ at all $t_{0}\left(0<t_{0}<1\right)$, then we say $f$ satisfies $P$ locally.

An interval $I \subseteq[0,1]$ is said to be stationary for $f$ if $f \mid I$ is a constant function. Our definition of neighborhood is necessitated by the presence of stationary intervals that are non-trivial (i.e., that have non-empty interior). The essential $f$-neighborhood of $t_{0}$ is the intersection of all $f$-neighborhood of $t_{0}$. The essential $f$-neighborhood of $t_{0}$ is stationary for $f$. In applications, we choose $X=\mathbb{R}^{2}$ with the Euclidean metric, and $f=F \mu$ where $\mu$ is any motion. Often, we want to focus on the properties of $\mu$, not $F \mu$. To do this, we use $F \mu$-intervals while discussing properties of $\mu$, as seen next. We define three local properties of motions:

- $\mu$ is locally straight. Let $P_{0}(\mu)$ be the property that "the trace $F \mu$ is straight". We say a motion $\mu$ is locally straight at $t$ if $P_{0}$ holds whenever $F$ is restricted to an $F \mu$-neighborhood of $t$.

- $\mu$ is locally a vertex. Let $P_{1}(\mu)$ be the property "there exists $0 \leq t \leq 1$ such that $\mu(t)$ is a vertex $\left(0\right.$-cell)". Then we say $\mu$ is locally a vertex at $t$ if $P_{1}$ holds 


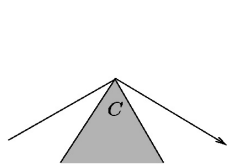

(a)

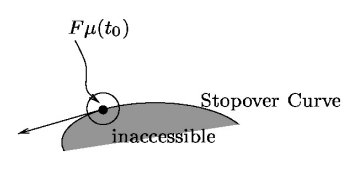

(b)

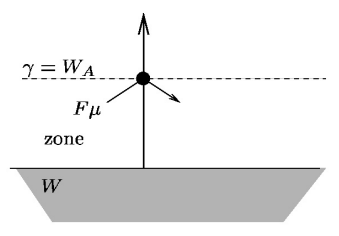

(c)

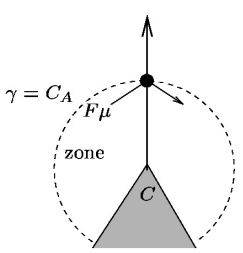

(d)

Fig. 2. Locally non-straight traces: (a) pivoting around corner, (b) tangential or tracing a stopover curve, (c),(d) reflecting off a displaced wall or corner.

whenever $\mu$ is restricted to an $F \mu$-neighborhood of $t$. Equivalently, $\mu$ is locally a vertex at $t$ iff $\mu(t)$ is a vertex for some $t$ in the $F \mu$-essential neighborhood of $t$.

- $\mu$ is locally reflecting. Let $P_{2}(\mu)$ be the property that "there exists $0 \leq t \leq 1$ such that $\mu(t)$ is reflecting". We say that $\mu$ is locally reflecting at $t$ if $P_{2}$ holds whenever $\mu$ is restricted to an $F \mu$-neighborhood of $t$.

Here is the statement of the main result:

Theorem 5 (Local Characterization). Let $\mu:[0,1] \rightarrow$ FP be a $d_{1}$-optimal motion and $0<t_{0}<1$. Suppose $\mu$ is not locally straight at $t_{0}$. Then one of the following four situations hold:

1. $\mu$ is locally a vertex at $t_{0}$.

2. $\mu\left(t_{0}\right)$ is pivotal at a convex corner $C$ (i.e., $\left.F\left[\mu\left(t_{0}\right)\right]=C\right)$. Moreover, $F \mu$ is locally "bending" around C. See Fig. 2(a).

3. $\mu\left(t_{0}\right)$ is constricted. Either (a) $F \mu$ is is locally tracing a stopover curve at $t_{0}$, or (b) the trace is a straight line that meets a stopover curve tangentially at $t_{0}$. See Fig. 2(b).

4. $\mu$ is locally reflecting at $t_{0}$. See Fig. 2(c),(d). Let the curve $\gamma$ be the displaced wall or corner where this reflection takes place. Then the trace F $\mu$ meets and leaves $\gamma$ at an incident point $r$ according to Snell's law. Morover, the trace in the neighborhood of $r$ lies within the zone of $\gamma$.

Note that this theorem does not say anything about the motion when $\mu$ is locally straight at $t_{0}$. In [3] we stated such a result without proof. We now provide the proof in Appendix III.

\section{Pseudo Approximation Algorithm for Rod Motion}

The main result of this section is the following.

Theorem 6. There is a pseudo approximation algorithm for $d_{1}$-optimal rod motion whose running time is $O\left(n^{5} \varepsilon^{-2}\left(n+\varepsilon^{-1}\right)\right)$.

Its proof follows the model of the 3ESP algorithm. We will define a fragmentation (depending on the input parameters $\left(Z_{0}, Z_{1}, \Omega\right), \varepsilon>0$ and $R \geq 0$ ) of the edges that 
bound the space of free configurations, and a "visibility graph" $F V G=(N, A ; W)$ on these fragments. Specifically, if $F P=F P(\Omega)$ is the space of free configurations, define the restricted free space to be $F P_{R}=\left\{Z \in F P: F[Z] \in \Omega_{R}\right\}$ where $\Omega_{R}$ is defined as before, namely $\Omega_{R}=\Omega \cap B(R)$ and $B(R)$ is the disk of radius $R$ centered at $F\left[Z_{0}\right]$. A restricted optimal motion of a rod is a motion $\mu_{R}^{*}:[0,1] \rightarrow F P_{R}$ such that $\mu_{R}^{*}(i)=Z_{i}$ $(i=0,1)$ and $d_{1}\left(\mu_{R}^{*}\right)$ is minimum.

To specify the node set $N$, we consider edges $e$ of the complex $\partial F P$ that are either stopover curves or mirrors. Let $e_{R}$ be the restriction of $e$ to $F P_{R}$. We can view such an edge $e$ as a motion $e:[0,1] \rightarrow F P$, and thus speak of the trace of $e$. We can subdivide $e$ into submotions, again called fragments. The length of a fragment $\sigma$ is simply the length of the trace $F \sigma$, denoted by $d_{1}(\sigma)$. The node set $N$ comprising these fragments, together with pivotal edges and $Z_{0}$ and $Z_{1}$, has total size $O\left(n^{5 / 2} \varepsilon^{\prime-1}\left(n^{1 / 2}+\varepsilon^{\prime-1 / 2}\right)\right)$. The fragmentation process depends on the type of the edge.

Straight Mirrors. We let each fragment have length $\leq \varepsilon^{\prime} R / n$. Each straight mirror is associated with a wall. Let $W$ be a wall of $F P_{R}$. Then $|W| \leq R$. Suppose $S_{W}$ comprise all mirror fragments associated with $W$. Then the total length of all the fragments in $S_{W}$ is $|W|$, and thus $\left|S_{W}\right| \leq n / \varepsilon^{\prime}$. Since there are $\leq n$ walls, the number of fragments from straight mirrors is $O\left(n^{2} / \varepsilon^{\prime}\right)$.

Stopover Edges. Again we first break the edges into fragments of length $\leq \varepsilon^{\prime} R / n$. Since every stopover edge has a total length which is $O(1)$, and there are $O\left(n^{2}\right)$ stopover edges, the total number of stopover fragments is $O\left(n^{3} / \varepsilon^{\prime}\right)$.

Circular Mirrors. This is more complicated. First, we create superfragments of length $\leq\left(\varepsilon^{\prime} / n\right)^{1 / 2}$. Each superfragment is further subdivided into fragments of length $\leq\left(\varepsilon^{\prime} / n\right)^{3 / 2}$. Each circular mirror is associated with a convex corner. Let $C$ be such a corner and let $S_{C}$ comprise all fragments associated with $C$. The length of all the fragments in $S_{C}$ is $<2 \pi$, and hence $\left|S_{C}\right|=O\left(\left(n / \varepsilon^{\prime}\right)^{3 / 2}\right)$. Since there are $\leq n$ corners, the number of fragments from circular mirrors is $O\left(n^{5 / 2} \varepsilon^{\prime-3 / 2}\right)$.

We define the arc set $A \subseteq N^{2}$ based on weak visibility again, but the low level computation is somewhat more complex (but $O(1)$ in the algebraic complexity model). As before the weight $W\left(\sigma_{a}, \sigma_{b}\right)$ of the arc joining weakly visible fragments $\sigma_{a}$ and $\sigma_{b}$ is taken to be the Euclidean distance between the fragment midpoints. In addition, there is an arc joining fragments from the same mirror superfragment or stopover edge whose length is just the distance along the mirror superfragment or stopover edge between the fragment midpoints. The connection between paths in the Fragment Visibility Graph and $d_{1}$-motion, through the analogues of $(11),(12)$, is complicated by the non-linear features of our domain, in particular circular mirrors.

Observation A. If $P$ is any single edge path in $F V G$ joining two fragments $\sigma_{a}$ and $\sigma_{b}$, not both of which are circular mirror fragments, then their exists a Euclidean path $\mu(P)$ in $\Omega_{R}$ joining their midpoints $\check{\sigma}_{a}$ and $\check{\sigma}_{b}$ such that $d(\mu(P)) \leq W(P)+\varepsilon^{\prime} R / n$.

Observation $\mathbf{A}^{\prime}$. If $P$ is any single edge path in $F V G$ joining two fragments $\sigma_{a}$ and $\sigma_{b}$ both of which are circular mirror fragments, then their exists a Euclidean path $\mu(P)$ in $\Omega_{R}$ joining their midpoints $\check{\sigma}_{a}$ and $\check{\sigma}_{b}$ such that $d(\mu(P)) \leq W(P)+\left(\varepsilon^{\prime} / n\right)^{3 / 2} R$. 
Observation B. If $P$ is any path in $F V G$ joining two fragments $\sigma_{a}$ and $\sigma_{b}$ of the same straight mirror or stopover edge, then their exists a Euclidean path $\mu(P)$ in $\Omega_{R}$ joining $\check{\sigma}_{a}$ and $\check{\sigma}_{b}$ such that $d(\mu(P)) \leq W(P)+\varepsilon^{\prime} R / n$.

Observation $\mathbf{B}^{\prime}$. If $P$ is any path in $F V G$ joining two fragments $\sigma_{a}$ and $\sigma_{b}$ of the same circular mirror superfragment, then their exists a Euclidean path $\mu(P)$ in $\Omega_{R}$ joining $\check{\sigma}_{a}$ and $\check{\sigma}_{b}$ such that $d(\mu(P)) \leq W(P)+\left(\varepsilon^{\prime} / n\right)^{3 / 2} R$.

Observation C. If a Euclidean line segment $\mu$ in $\Omega_{R}$ joins two points $v_{a}$ and $v_{b}$, belonging to fragments $\sigma_{a}$ and $\sigma_{b}$ not both of which are circular mirror fragments, then there exists a path $P(\mu)$ in $F V G$ joining fragments $\sigma_{a}$ and $\sigma_{b}$ such that $W(P(\mu)) \leq$ $d(\mu)+\varepsilon^{\prime} R / n$.

Observation $\mathbf{C}^{\prime}$. If a Euclidean line segment $\mu$ in $\Omega_{R}$ joins two points $v_{a}$ and $v_{b}$, belonging to fragments $\sigma_{a}$ and $\sigma_{b}$, both of which are circular mirror fragments, then there exists a path $P(\mu)$ in $F V G$ joining fragments $\sigma_{a}$ and $\sigma_{b}$ such that $W(P(\mu)) \leq$ $d(\mu)+\left(\varepsilon^{\prime} / n\right)^{3 / 2} R$.

Observation D. If a Euclidean path $\mu$ in $\Omega_{R}$ joins two points $v_{a}$ and $v_{b}$, belonging to fragments $\sigma_{a}$ and $\sigma_{b}$ of the same straight mirror or stopover edge, then there exists a path $P(\mu)$ in $F V G$ joining fragments $\sigma_{a}$ and $\sigma_{b}$ such that $W(P(\mu)) \leq d(\mu)+\varepsilon^{\prime} R / n$.

Observation D'. If a Euclidean path $\mu$ in $\Omega_{R}$ joins two points $v_{a}$ and $v_{b}$, belonging to fragments $\sigma_{a}$ and $\sigma_{b}$ of the same straight mirror superfragment, then there exists a path $P(\mu)$ in $F V G$ joining fragments $\sigma_{a}$ and $\sigma_{b}$ such that $W(P(\mu)) \leq d(\mu)+\left(\varepsilon^{\prime} / n\right)^{3 / 2} R$.

Let $P$ be any path in the $F V G$ from $Z_{0}$ to $Z_{1}$. We can decompose $P$ into subpaths $P_{1}, P_{2}, \ldots, P_{k}$, where each $P_{i}$ is either (i) a single edge joining fragments of distinct stopover edges, straight mirrors, or circular mirror superfragments, or (ii) maximal subpaths joining fragments of the same stopover edge, straight mirror or circular mirror superfragment. By maximality each stopover edge, straight mirror or circular mirror superfragment can be involved in at most two subpaths of type (i). Thus there are $O(n)$ subpaths of type (i) involving at least one non-circular mirror fragment, and $O\left(n\left(n / \varepsilon^{\prime}\right)\right)$ subpaths of type (i) joining circular mirror fragments. Similarly, there are $O(n)$ subpaths of type (ii) involving at least one non-circular mirror fragment, and $O\left(n\left(n / \varepsilon^{\prime}\right)\right)$ subpaths of type (ii) joining circular mirror fragments. If we define $\mu(P)$ to be the Euclidean path formed by concatenation of $\mu\left(P_{1}\right), \ldots, \mu\left(P_{k}\right)$, then, by Observations $\mathrm{A}, \mathrm{A}^{\prime}, \mathrm{B}$ and $\mathrm{B}^{\prime}$, we have

$$
d\left(\mu(P) \leq W(P)+O\left(\varepsilon^{\prime} R\right) .\right.
$$

Similarly, if $\mu$ is any Euclidean path in $\Omega_{R}$, then $\mu$ can be decomposed into subpaths $\mu_{1}, \mu_{2}, \ldots, \mu_{k}$ such that each $\mu_{i}$ is either (i) a single line segment joining fragments of distinct stopover edges, straight mirrors, or circular mirror superfragments, or (ii) a maximal subpath joining fragments of the same stopover edge, straight mirror or circular mirror superfragment. 
Define $P(\mu)$ to be the path in $F V G$ formed by concatenation of the paths $P\left(\mu_{1}\right), \ldots$, $P\left(\mu_{k}\right)$. By Observations $\mathrm{C}, \mathrm{C}^{\prime}, \mathrm{D}$ and $\mathrm{D}^{\prime}$, we have

$$
W(P(\mu)) \leq d(\mu)+O\left(\varepsilon^{\prime} R\right)
$$

We now put these two steps together. Begin with the shortest path $P^{*}$ joining $Z_{0}$ and $Z_{1}$ in $F V G$. Using the construction above, we obtain a Euclidean path $\mu\left(P^{*}\right)$. As before it follows directly from (13) and (14) that $d\left(\mu\left(P^{*}\right)\right) \leq d\left(\mu_{R}^{*}\right)+O\left(\varepsilon^{\prime} R\right)$.

This concludes our proof of Theorem 6 . Now we can apply the geometric search algorithm to obtain a true $\varepsilon$-approximation algorithm whose running time depends on $\lg \left(1 / d_{1}\left(\mu^{*}\right)\right)$. We next prove a lower bound on $d_{1}\left(\mu^{*}\right)$.

\section{Lower Bound on Non-Zero $d_{1}\left(\mu^{*}\right)$}

This section is devoted to the proof of the following result:

Theorem 7. Assume $d_{1}\left(\mu^{*}\right)>0$. Then $d_{1}\left(\mu^{*}\right) \geq c^{-L}$ for some constant $c>1$.

First, recall that the input description, including all obstacle corners and the initial position $F\left[Z_{0}\right]$ of the rod focus point $F$, involves only $L$-bit rational numbers. We assume that the lengths of the half-rods, $A F$ and $F B$, are also $L$-bit rational numbers, denoted $a$ and $b$, respectively.

The sparsity of points with small rational coordinates allows us to dispose easily of the case in which the initial and final positions of the focus differ: if $F\left[Z_{0}\right] \neq F\left[Z_{1}\right]$, then $d_{1}\left(Z_{0}, Z_{1}\right) \geq\left\|F\left[Z_{0}\right]-F\left[Z_{1}\right]\right\| \geq 2^{-2 L}$. Henceforth, assume $F\left[Z_{0}\right]=F\left[Z_{1}\right]$. In this case the theorem is an immediate consequence of the following:

Main Claim. If $F[Z]$ is never too far (no further than $c^{-L}$, for some fixed constant $c>1)$ from $F\left[Z_{0}\right]$, then the placements $F\left[Z_{0}\right]$ and $F\left[Z_{1}\right]$ must be equivalent up to a pure rotation (i.e., $\left.d_{1}\left(Z_{0}, Z_{1}\right)=0\right)$.

Imagine rotating the rod about its focus $F$. We refer to the first obstacle feature encountered in a clockwise (respectively, counterclockwise) rotation in configuration $Z$ as the $\mathbf{C W}$ (respectively, $\mathbf{C C W}$ ) feature stop at $Z$. Two configurations with coincident foci are equivalent up to a pure rotation if and only if their $\mathrm{CW}$ and $\mathrm{CCW}$ feature stops are identical. To establish this equivalence it suffices to consider each rod endpoint separately and show that the $\mathrm{CW}$ and $\mathrm{CCW}$ feature stops of each half-rod are identical in the two configurations.

The argument will be made for the half-rod $A F$; the corresponding argument for $F B$ is identical. Start by considering an open disk $D$ of radius $a$ centered at $F\left[Z_{0}\right]$. Then every obstacle wall either (i) does not intersect $D$; (ii) intersects the boundary of $D$ twice (and so has no associated corner in $D$ ); (iii) intersects the boundary of $D$ once (and so has exactly one corner in $D$ ); or (iv) is entirely contained (and so has both of its associated corners) in $D$. We choose representative points associated with walls as follows: Walls of type (i) have no representative point. Walls $w$ of type (ii) are represented 
by their closest point $p_{w}$ to $F\left[Z_{0}\right]$, provided $p_{w} \neq F\left[Z_{0}\right]$. (If $p_{w}=F\left[Z_{0}\right]$, then $w$ has no representative point.) Walls $w$ of type (iii) are represented by their corner inside $D$, provided this corner does not coincide with $F\left[Z_{0}\right]$. (If the corner coincides with $F\left[Z_{0}\right]$, then $w$ has no representative point.) Finally, walls $w$ of type (iv) are represented by their two associated corners, provided the corner does not coincide with $F\left[Z_{0}\right]$.

Walls of type (ii) and (iii) that do not have representative points (because they pass through or end at $F\left[Z_{0}\right]$ ) serve to partition $D$ into wedges centered at $F\left[Z_{0}\right]$. The wedge occupied by the rod in its initial configuration (the entire disk if there are no edges of type (ii) or (iii)) is called the primary wedge. Clearly, unless $F[Z]$ exceeds distance $a$ from $F\left[Z_{0}\right]$ somewhere in its trace, the rod also occupies the primary wedge in its final configuration. The representative points serve to partition the primary wedge of $D$ into secondary wedges formed by rays centered at $F[Z]$ through the individual representative points. Note that as $Z$ changes the secondary wedges change. However, if $F[Z]$ never leaves a disk of radius $c^{-L}$, where $c>1$ is a fixed constant, then (i) the distance from $F[Z]$ to individual representative points never exceeds $a$, and (ii) the relative order of the rays defining the secondary wedges (except for those rays that coincide in the initial, and final, configuration) remains fixed. This follows from the following elementary observations:

Observation A. All representative points are described by $O(L)$-bit rational numbers.

Observation B. The distance from $F\left[Z_{0}\right]$ to its closest point on the radius $a$ circle centered at any representative point is at least $c^{-L}$, for some positive constant $c>1$.

Observation C. The distance from $F\left[Z_{0}\right]$ to its closest point on the line joining any two representative points is either zero or at least $c^{-L}$, for some positive constant $c>1$.

It follows that the secondary wedges, $\Delta_{0}$ and $\Delta_{1}$, occupied by the half-rod $A F$ in its initial and final configurations are idential. It remains to show that any free placement $Z$ of $A F$ in $\Delta_{0}$ with $F[Z]=F\left[Z_{0}\right]$ has a unique CW and CCW stop. Since $\Delta_{0}$ contains no representative points in its interior, if the $\mathrm{CW}$ (or $\mathrm{CCW}$ ) stop of $A F$ at $Z$ is realized by an obstacle corner, then that corner must be a representative point defining one of the bounding rays of $\Delta_{0}$. If the $\mathrm{CW}$ (or $\mathrm{CCW}$ ) stop of $A F$ at $Z$ is realized by a wall $w$, we observe that: (i) since $A F$ has a free placement in $\Delta_{0}, w$ does not intersect both sides of $\Delta_{0}$; (ii) since $\Delta_{0}$ contains no representative points in its interior, $w$ does not have an associated corner inside $\Delta_{0}$, or cross the boundary of $D$ twice within $\Delta_{0}$; and hence (iii) $w$ must intersect the boundary of $D$ and one of the sides of $\Delta_{0}$. The uniqueness of $w$ follows from the fact that obstacle walls do not intersect.

This concludes the proof of the Main Claim.

\section{Final Remarks and Open Problems}

This paper has demonstrated the usefulness of pseudo approximation algorithms. Applied to two of the simplest $N P$-hard optimum motion planning problems, we obtain new $\varepsilon$ - 
approximation algorithms. Many interesting questions remain to be explored:

(1) There should be other applications of our general methodology to exploiting pseudo approximations.

(2) Our complexity analysis is in the algebraic model of computation. It is of interest to obtain true bit-complexity bounds, in the spirit of Choi et al. [7].

(3) The complexity of the two approximation algorithms (shortest path for a point robot in three dimensions and $d_{1}$-shortest path for a rod in two dimensions) should be possible to improve, by not treating the pseudo approximation algorithms as black-boxes in the binary search scheme.

(4) Can we extend these techniques to approximating general $d_{n}$-optimal motion, and also to an arbitrary rigid planar robot?

\section{Acknowledgements}

The authors thank John Hershberger for his many insightful comments on the paper. The Binary Searches of Section 2 are improved from the original proceedings, thanks to an insightful question from David Mount about the necessity of a $\log (1 / \varepsilon)$ term in our original complexity bound.

\section{Appendix I. Basic Vocabulary}

A rod is a closed line segment $\overline{A B}$, directed from the $B$-end (base) to the $A$-end (apex). The $A$-end is the end with the arrow head in figures. See Fig. 1(a). So we also think of the rod as a line segment, directed from the $B$-end to the $A$-end. The corresponding open segment is simply denoted as $A B$. The focus is a point $F$ in the relative interior of the rod. The rod is thereby divided into two half-rods, $A F$ and $B F$, viewed as open segments.

The closure and boundary of an arbitrary set $S \subseteq \mathbb{R}^{2}$ is denoted by $\bar{S}$ and $\partial S$, respectively. We are given a closed planar set $\Omega \subseteq \mathbb{R}^{2}$ in which the rod is free to move. Its boundary $\partial \Omega$ is polygonal, and is naturally partitioned into pairwise disjoint sets: singleton sets called corners and open line segments called walls. An obstacle feature refers to either a corner or a wall. The closure $\bar{W}$ of an open wall $W$ is called a closed wall. For simplicity, we assume non-degeneracy on $\Omega$ as convenient.

We use the language of "placements" [25]. A placement is a pair $Z=(p, \theta) \in \mathbb{R}^{2} \times \mathbb{S}^{1}$ where $p \in \mathbb{R}^{2}$ is a point and $\theta \in \mathbb{S}^{1}$ is an angle. We also write $Z=(x, y, \theta)$ if $p=(x, y)$. For any set $S \subseteq \mathbb{R}^{2}$, we write $S[Z] \subseteq \mathbb{R}^{2}$ for the position of the set $S$ in placement $Z$. This position $S[Z]$ is obtained by rotating the plane containing $S$ about the origin by $\theta$, then translating the plane by $p$, viewed upon some reference plane. For example, $\overline{A B}[Z]$ is the position of the rod in placement $Z$, and it is just a closed line segment. Thus the symbol " $[Z]$ " acts as a Euclidean transformation of the plane. We choose a canonical representation of our $\operatorname{rod} A B: F=(0,0), A=(-a, 0)$ and $B=(b, 0)$ for some $0<a<1$ and $b=1-a$. For placement $Z=(x, y, \theta)$, we have $F[Z]=(x, y)$, $A[Z]=(x-a \cos \theta, y-a \sin \theta)$ and $B[Z]=(x+b \cos \theta, y+b \sin \theta)$. Furthermore,

$$
\overline{A B}[Z]=\{(x+t \cos \theta, y+t \sin \theta):-a \leq t \leq b\} .
$$


A placement $Z$ is free if $\overline{A B}[Z] \subseteq \Omega$. Let $F P=F P(\Omega)$ denote the set of free placements (or configuration space). Consider a continuous function

$$
\mu:[s, t] \rightarrow \mathbb{R}^{2} \times \mathbb{S}^{1},
$$

where $[s, t]$ is a real interval. For any point $X \in \mathbb{R}^{2}$, let $X \mu:[s, t] \rightarrow \mathbb{R}^{2}$ denote the path $X \mu(t)=X[\mu(t)]$. We call $X \mu$ the $X$-trajectory of $\mu$. In case $X=F$ (the focus of the rod), the $X$-trajectory is called the trace of $\mu$. We call $\mu$ a potential motion if both its $A$-trajectory and $B$-trajectory are rectifiable (i.e., has a definite arc length). This implies the trace of $\mu$ has a length $d_{1}(\mu)$, called the $d_{1}$-distance of $\mu$. A potential motion $\mu$ is a feasible motion (or simply "motion") if $\overline{A B}[\mu(u)] \subseteq \Omega$ for all $u \in[s, t]$. The $d_{1}$-distance between $Z, Z^{\prime} \in F P$, denoted by $d_{1}\left(Z, Z^{\prime}\right)$, is the minimum $d_{1}$-distance of a feasible motion from $Z$ to $Z^{\prime}$. A motion $\mu$ is $d_{1}$-optimum if $d_{1}(\mu)=d_{1}(\mu(0), \mu(1))$.

For $r \geq 0$ and $p \in \mathbb{R}^{2}$, let $B_{r}(p)$ denote the closed Euclidean ball of radius $r$ centered at $p$. Write $B_{r}(0)$ when $p$ is the origin. For $S \subseteq \mathbb{R}^{2}$, let $B_{r}(S)$ denote the set $\bigcup\left\{B_{r}(p): p \in S\right\}$ (alternatively, $B_{r}(S)$ is the Minkowski sum $\left.B_{r}(0) \oplus S\right)$. For a free placement $Z, B_{r}(A B[Z])$ has the shape of a capsule or "racetrack". The clearance Clearance $(Z)$ is defined to be the largest $r \geq 0 \operatorname{such} B_{r}(A B[Z]) \subseteq \Omega$. Let the racetrack of $Z$ refer to the set $B_{r}(A B[Z])$ when $r=h(Z)$; we denote the racetrack of $Z$ by $R T(Z)$. We say $Z$ is closest to those obstacle features $s$ that intersect $R T(Z)$ on its boundary. The racetrack shown in Fig. 3 shows two closest features, $s_{1}, s_{2}$. By definition, the interior of a racetrack has no obstacle points.

\section{Appendix II. Structure of $\partial F P$}

We focus on the boundary $\partial F P$ of $F P$ : $\partial F P$ comprise those placements $Z$ such that $\overline{A B}[Z]$ touches at least one obstacle feature. That is, $Z \in \partial F P$ iff there exists a wall $W$ or a convex corner $C$ such that at least one of three conditions hold: $A[Z] \in W$ or $B[Z] \in W$ or $C \in A B[Z]$. We will partition $\partial F P$ into a cell complex.

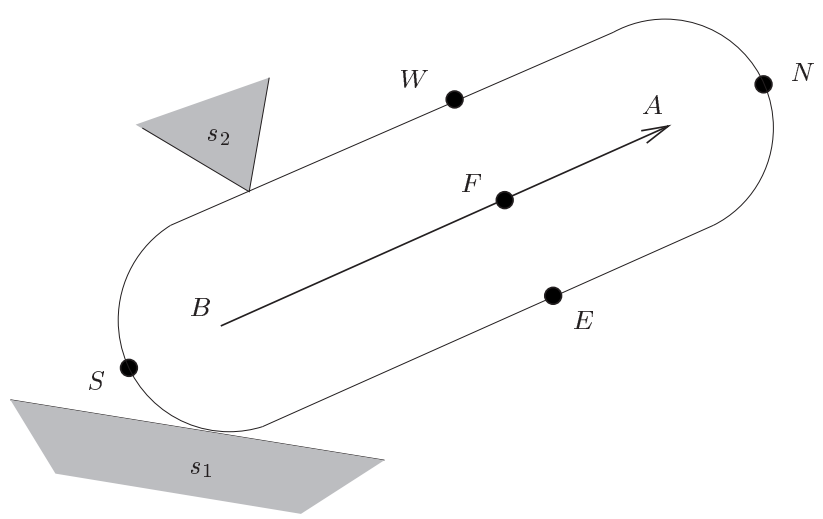

Fig. 3. Racetrack $R T(Z)$ : closest features $s_{1}, s_{2}$ and partition of its boundary. 


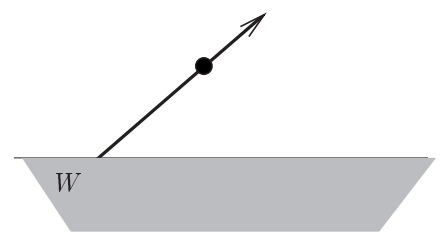

(a) $\left[B^{+} @ W\right]$

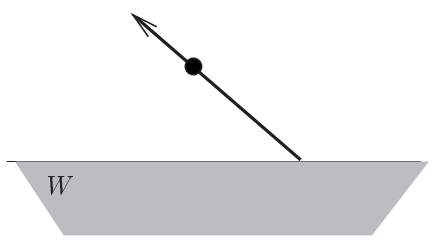

(b) $\left[B^{-@ W]}\right.$

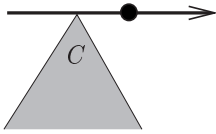

(c) $\left[B^{+} @ C\right]$

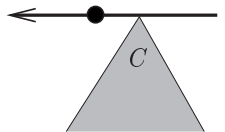

(d) $\left[B^{-@} C\right]$

Fig. 4. Basic constraints.

Constraints. We describe the cell complex via the intermediate concept of constraints (the treatment here is slightly different from [3]). First, consider four types of basic constraints, denoted as

$$
\left[B^{+} @ W\right],\left[B^{-} @ W\right],\left[B^{+} @ C\right],\left[B^{-} @ C\right] .
$$

We could replace $B$ by $A$ to get four more basic constraints. Altogether there are eight types of basic constraints. We emphasize that the corners $C$ in constraints are always convex corners, i.e., the obstacle set $\mathbb{R}^{2} \backslash \Omega$ is locally convex at $C$.

An obstacle feature $s$ is a clockwise stop (CW stop) for $Z$ if a $C W$ rotation of the rod about the focus $F$, starting at $Z$, first becomes infeasible by virtue of crossing $s$. A counterclockwise stop (CCW stop) for $Z$ is similarly defined. According to this definition, if an endpoint of a rod just grazes a feature $s$, then $s$ is not considered a stop. For instance, in Fig. 4(a), $W$ is a CCW stop for the indicated placement $Z$.

Constraints are properties of placements. A placement $Z$ with a constraint property $\xi$ is said to satisfy that $\xi$, and written $Z \models \xi$. For instance, the constraint $\left[B^{+} @ W\right]$ (read " $B$ counterclockwise at $W$ ") is satisfied by placement $Z$ if $B[Z] \in W$ and $W$ is a CCW stop for $Z$ (see Fig. 4(a)). Similarly, $\left[B^{-} @ C\right.$ ] (" $B$ clockwise at $C$ ") is satisfied by $Z$ if $C \in B F[Z]$ and $C$ is a CW stop for $Z$ (see Fig. 4(d)). The other two basic constraints are illustrated in Fig. 4(b),(c).

Recall that $B F$ is regarded as an open segment in this definition. Similarly, $W$ is an open segment. A consequence of this definition is that if $B[Z]=C$, then $Z$ does not satisfy any of the basic constraints. This is by design. 
Patches, Edges, Vertices. For any basic constraint $\xi$, the set $\{Z \in F P: Z \models \xi\}$ is seen to be relatively open. Each connected component of this set is a two-dimensional cell. The closure of such a cell is called a $\xi$-patch. Next, we define the one-dimensional cells, also known as edges of FP. These are the connected components of the intersection of two patches. Again, we can use the intermediate concept of constraints: an edge constraint is any pair of basic constraints $\xi_{1}, \xi_{2}$, which we write as $\xi_{1} \wedge \xi_{2}$. We say $Z$ satisfies the edge constraint, $Z \models \xi_{1} \wedge \xi_{2}$, if ${ }^{2} Z$ lies in the $\xi_{1}$-patch and the $\xi_{2}$ patch. For instance, $\left[B^{-} @ C\right] \wedge\left[A^{+} @ W\right]$ is an edge constraint satisfied by $Z$ such that $C \in B F[Z], A \in W[Z]$ and for which $C$ and $W$ are $C W$ and CCW stops, respectively. If $Z$ satisfies an edge constraint that has $\mathrm{CW}$ and $\mathrm{CCW}$ stops, we say $Z$ is constricted. Finally the intersection of three or more independent patches is a zero-dimensional set. Each placement in such a set is called a vertex.

Note that by definition, patches and edges are closed sets: an edge contains vertices for its endpoints, and a patch contains edges for its boundary. Let an open patch be defined as a patch minus its boundary edges, and an open edge be an edge minus its endpoints. Then the open patches, open edges and vertices constitute a 2-complex for $\partial F P$.

The classification of edges and vertices is more involved than for patches. Note that in [3], we gave a somewhat different constraint analysis; in particular, the "edges" there do not coincide with the ones used here. However, for our present purposes, it is enough to introduce three special classes of edges: mirrors, stopovers and pivots. Informally, the kind of edges excluded from this list are those satisfying an edge constraint which involve either two CW stops or two CCW stops.

Mirrors and Displaced Features. There are two kinds of mirrors: straight mirrors and circular mirrors. A placement $Z$ of a mirror is said to be reflecting. The case of straight mirrors is easy to characterize: a straight mirror is an edge of $\partial F P$ that satisfies a joint constraint $\left[X^{+} @ W\right] \wedge\left[X^{-} @ W\right]$, where $X=A$ or $B$ and $W$ is any wall. Figure 5(a) shows a wall $W$ giving rise to two straight mirrors. However, in general, due to the presence of other obstacles, a wall can give rise to a linear number of mirrors.

Corresponding to each mirror is a natural mirror motion; the trace of this motion is straight or circular, and is called a displaced wall feature $\left(W_{A}, W_{B}\right.$ in Fig. 5(a)) or a displaced corner feature $\left(C_{A}, C_{B}\right.$ in Fig. 5(b)). These displaced features are shown as dashed lines. Furthermore, the portion of the plane between the wall $W$ and the displaced feature $W_{X}(X=A, B)$ that is swept by the half-rod $F X$ is called the zone of the mirror.

Analysis of Circular Mirrors. We want to analyze the nature of the circular mirrors associated with a convex corner $C$. This analysis will also help clarify our decomposition of $\partial F P$ based on patches. Consider the exterior angle at $C$, bounded by the incident walls $W_{1}, W_{2}$ (see Fig. 5(c),(d)). This angle is partitioned into five sectors, by extending the walls $W_{1}, W_{2}$ into the exterior angle, and by introducing normals to these walls at $C$. The extension and normal of $W_{i}$ are denoted $W_{i}^{\text {ext }}$ and $W_{i}^{\perp}$ in Fig. 5(c),(d).

\footnotetext{
${ }^{2}$ It important to realize that this is not the same as saying $Z \models \xi_{1}$ and $Z \models \xi_{2}$.
} 


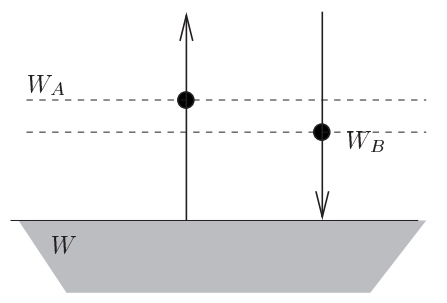

(a)

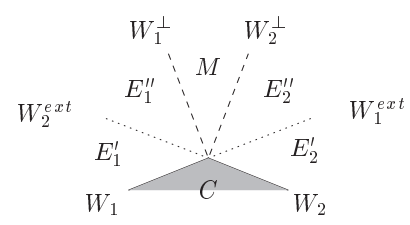

(c)

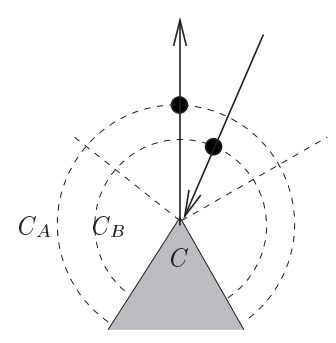

(b)

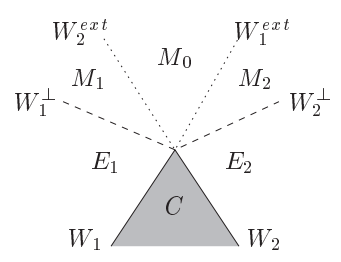

(d)

Fig. 5. (a),(b) Mirrors and displaced features. (c),(d) Analysis of circular mirrors.

Let $M$ denote the sector bounded by the two normals; the rest of the exterior angle has two connected parts denoted $E_{1}$ and $E_{2}$. Let $F P_{A}(C)=\{Z \in F P: A[Z]=C, \overline{A B}[Z] \in$ $M\}$ (there is a similar $F P_{B}(C)$ ). If $Z \in F P_{A}(C)$, we say $Z$ belongs to a sector, say $M$, if $A B[Z]$ is contained in $M$, etc. We consider two cases, depending on whether the angle at $C$ is greater than (Case (c)) or less than (Case (d)) a right angle.

- Case (c). In this case, $E_{i}$ is in turn divided into two parts $E_{i}^{\prime}$ and $E_{i}^{\prime \prime}(i=1,2)$, where $E_{i}^{\prime}$ is the part adjacent to the wall $W_{i}$. Referring to Fig. 5(c), note that a placement $Z \in F P_{A}(C)$ belongs to $E_{1}^{\prime}$ iff

$$
Z \models\left[A^{-} @ W_{1}\right] \wedge\left[A^{-} @ C\right] .
$$

Also, $Z$ belongs to $E_{1}^{\prime \prime}$ iff

$$
Z \models\left[A^{-} @ W_{1}\right] \wedge\left[A^{-} @ W_{2}\right] .
$$

We replace $W_{1}$ by $W_{2}$ and $A^{-}$by $A^{+}$in case of $E_{2}^{\prime}$ and $E_{2}^{\prime \prime}$. Finally, $Z$ belongs to $M$ iff

$$
Z \models\left[A^{-} @ W_{1}\right] \wedge\left[A^{+} @ W_{2}\right] .
$$

- Case (d). In this case, $M$ is divided into three parts named $M_{1}, M_{0}, M_{2}$. Referring to Fig. 5(d), note that a placement $Z \in F P_{A}(C)$ belongs to $E_{1}$ iff

$$
Z \models\left[A^{-} @ W_{1}\right] \wedge\left[A^{-} @ C\right] .
$$

In the case of $E_{2}$, we replace $A^{-}$by $A^{+}$and $W_{1}$ by $W_{2}$. Next, $Z$ belongs to $M_{1}$ iff

$$
Z \models\left[A^{+} @ W_{1}\right] \wedge\left[A^{-} @ C\right] .
$$


Similarly, $Z$ belongs to $M_{2}$ iff

$$
Z \models\left[A^{-} @ W_{2}\right] \wedge\left[A^{+} @ C\right] .
$$

Finally, $Z$ belongs to $M_{0}$ iff

$$
Z \models\left[A^{+} @ W_{1}\right] \wedge\left[A^{-} @ W_{2}\right] .
$$

Summarizing cases (c) and (d), we can say $Z \in F P_{A}(C)$ belongs to $M$ iff $Z$ satisfies a joint clockwise $\left(A^{-}\right)$and counterclockwise $\left(A^{+}\right)$constraint.

Of course, the presence of other obstacles breaks up $F P_{A}(C)$ into several connected components. Each connected component in $F P_{A}(C)$, restricted to one of the five sectors constitutes an edge of $\partial F P$. By definition, a circular mirror is one of these edges comprising placements that belong to $M$. The boundary between these sectors, when they are free, are vertices of $\partial F P$.

The terminology of "mirrors" comes from the fact that the trace of optimum motions sometimes "reflect" off the displaced wall or corner, the reflection obeying the law of reflection. This mirror phenomenon is critical for our NP-hardness proof in [3].

Stopover Edges and Stopover Curves. We define a stopover edge as a constricted edge whose trace is not straight. Recall that $Z \in \partial F P$ is constricted iff $Z$ has a CW stop and a CCW stop, so it cannot rotate. The trace of a stopover edge is called a stopover curve. There are three kinds of stopover edges, and their constraints are:

(1) $\left[A^{+} @ W_{1}\right] \wedge\left[B^{-} @ W_{2}\right]$ where $W_{1}, W_{2}$ are non-parallel walls: the trace is part of an ellipse.

(2) $\left[A^{+} @ W\right] \wedge\left[X^{-} @ C\right]$ where $X=A$ or $B$ : the trace is part of a conchoid.

(3) $\left[A^{+} @ W_{1}\right] \wedge\left[A^{-} @ W_{2}\right]$ : this can only happen when the $A$-end of the rod is (stuck) at a concave corner determined by $W_{1}$ and $W_{2}$. The trace is a circular arc.

Of course, in the above constraints, we could also interchange the roles of $A$ and $B$. The last kind of constricted edge, which we do not consider to be a stopover edge, satisfies the constraints $\left[A^{+} @ C_{1}\right] \wedge\left[B^{-} @ C_{2}\right]$ or $\left[A^{+} @ C_{1}\right] \wedge\left[A^{-} @ C_{2}\right]$ or where $C_{1}, C_{2}$ are corners. The trace is a straight line segment.

Pivotal Edges. The third class of edges is very simple: they satisfy the pivotal constraint $\left[A^{+} @ C\right] \wedge\left[B^{-} @ C\right]$ where $C$ is any convex corner (the roles of $A, B$ can be exchanged). Note that $Z$ satisfies this constraint iff $F[Z]=C$. We call $Z$ pivotal in this case. A maximal connected set of such placements is a pivotal edge and corresponds to a motion of pure rotation about the focus which is fixed at some $C$.

Complexity of the Cell Complex. We conclude this appendix by bounding the size of the cell complex of $\partial F P$ which we just described.

Theorem 8. Let there be $n$ wall and corner features. In our 2-complex of $\partial F P$, the following bounds hold:

(a) The number of patches is $O\left(n^{2}\right)$. 
(b) The number of edges is $O\left(n^{3}\right)$.

(c) The number of vertices is $O\left(n^{3}\right)$.

Proof. (a) Each patch is determined by one of the $8 n$ basic constraints. A basic constraint $\xi$ can give rise to $<n$ patches. To see this suppose the constraint $\xi$ relates to a wall $W$, say $\xi=\left[A^{+} @ W\right]$. In the absense of any other features there is only one patch. We now introduce features one at a time: each feature can only increase the number of patches by one. First we introduce the corner features. Each corner feature, if it increases the number of patches, can only do so by splitting one current patch into two new patches. Now we introduce the wall features (their corners have already been introduced). However, these cannot increase the number of patches. A similar argument applies when $\xi$ relates to a corner $C$, as in $\xi=\left[A^{+} @ C\right]$.

(b) There are $64 n^{2}$ edge constraints. Each edge constraint $\xi_{1} \wedge \xi_{2}$, in the absence of any other features, determines a one-dimensional set of placements that is a connected set. Again, each new feature we introduce, if it disrupts the connectivity of any connected component, can only increase the number of components by one. Hence there are at most $64 n^{3}$ edges in the complex.

(c) Each vertex can be charged to a triple of obstacle features. There are $n^{3}$ such triples. However, every triple can be charged a constant number of times. Hence the number of charges is $O\left(n^{3}\right)$.

\section{Appendix III. Proof of the Local Characterization Theorem}

We prove Theorem 5 which gives a local characterization the $d_{1}$-optimal motion.

The following classification of placements is based on considering the behavior of the clearance function as we make small rotatations, where rotations are always about the focus $F$ :

\section{Definition 1.}

(1) The placement $Z$ is stopped if Clearance $(Z)$ is locally maximum in the sense that any infinitesimal rotation $(\mathrm{CW}$ or $\mathrm{CCW})$ will decrease the clearance.

(2) A placement $Z$ is critical if Clearance $(Z)$ is locally minimum in the sense that any infinitesimal rotation $(\mathrm{CW}$ or $\mathrm{CCW})$ will increase the the clearance.

(3) Define a rotation function $\rho: F P \rightarrow F P$ as follows. If $Z$ is stopped or critical, let $\rho(Z)=Z$. Otherwise, there is a unique direction to rotate $Z$ so as to increase the clearance of $Z$ locally. Define $\rho(Z)$ to be the first local maxima reached by this rotation. We also define

$$
H(Z)=\text { Clearance }(\rho(Z)) .
$$

In Appendix I we introduced the racetrack $R T(Z)$. See Fig. 3. If Clearance $(Z)>0$, then the boundary of $R T(Z)$ contains four special points called the North, South, East, West poles of $Z$. The boundary is thereby divided into four open curves, called the North-East, North-West, South-East, South-West tracks. Stopped placements can be characterized as placements whose East or West poles are covered, or two adjacent tracks 
are covered. (Two tracks are adjacent if they are both bounded by one of the four poles, e.g., the North-East track is adjacent to North-West and to South-East tracks.) Similarly, we may characterize critical placements to be those placements $Z$ whose North or South poles are "covered" (i.e., contained in some obstacle feature), and, furthermore, no point on the boundary of $R T(Z)$ is covered. The latter condition is important, as it implies that a critical placements could not be simultaneously stopped.

These characterizations depend upon the assumption Clearance $(Z)>0$, but they can be extended to the case Clearance $(Z)=0$ by taking limits. Thus, stopped placements and critical placements are generalizations (respectively) of constricted placements and reflecting placements. The requirement that critical placements must not have any other points on its racetrack boundary covered is translated into the requirement that a reflecting placement must not satisfy any other constraints (other than what is required by definition).

Next we introduce some special motions. Let $\mu:[0,1] \rightarrow F P$ be a motion.

- $\mu$ is constricted if for all $t \in[0,1], \mu(t)$ is constricted.

- $\mu$ is pivotal if for all $t \in[0,1], \mu(t)$ is pivotal. In this case there is a unique convex corner $C$ such that $F \mu(t)=C$.

- $\mu$ is reflecting if for all $t \in[0,1], \mu(t)$ is reflecting.

- $\mu$ is restrained if it is constricted, pivotal or reflecting.

- A placement $Z$ is restrained if it is one of the following: (a) constricted, (b) pivotal, (c) reflecting or (d) a vertex. Remark: Clause (d) might seem unnecessary, but Fig. 6(i) shows a vertex $V_{1}$ that does not fall under (a), (b) or (c). We now prove two lemmas about unrestrained placements. More important is the remark that our definition of a reflecting placement $Z$ does not allow the $Z$ to satisfy any other constraints. Thus the vertices $V_{2}$ and $V_{3}$ in Fig. 6(ii),(iii) are also not covered by clauses (a), (b) or (c). In proofs we do not care if the vertex $V_{1}$ is considered restrained (though it is harmless to be considered restrained); but we do need $V_{2}, V_{3}$ to be considered restrained.

- A motion $\mu$ is unrestrained if for all $t, 0<t<1, \mu(t)$ is not restrained. Note $\mu(0)$ and $\mu(1)$ may be restrained in this definition.

- $\mu$ is straight if for all $t \in[0,1], F \mu(t)$ lies on the straight line segment $[F \mu(0), F \mu$ (1)]. Moreover, the trace is monotone in the sense that for $0 \leq t<t^{\prime} \leq 1, F \mu\left(t^{\prime}\right)$ is closer than $F \mu(t)$ to $F \mu(1)$.

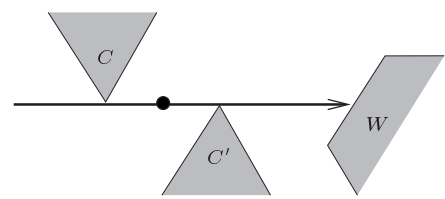

(i) $V_{1}$

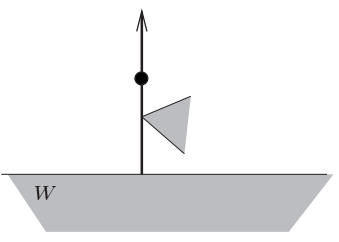

(ii) $V_{2}$

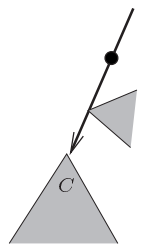

(iii) $V_{3}$

Fig. 6. Vertices that are not constricted, pivotal or reflecting. 
Lemma 9. If $Z$ is unrestrained, then $H(Z)>0$.

Proof. If Clearance $(Z)>0$, then the result follows from the fact that $H(Z) \geq$ Clearance $(Z)$. So assume Clearance $(Z)=0$. In this case $\overline{A B}[Z]$ must touch some feature $s$ (wall or corner). There is a unique direction in which we rotate $Z$ away from $s$; this is because $s$ cannot be a corner at $F[Z]$ (it would make $Z$ pivotal), and $s$ is not a wall normal to $A B[Z]$ (it would make $Z$ reflecting). Moreover, if we make a small enough rotation, $Z$ would remains free (otherwise $Z$ is constricted). This proves that $\rho(Z) \neq Z$, i.e., $H(Z)>0$.

Lemma 10. Let $\mu:[0,1] \rightarrow F P$ be an unrestrained motion. If $\mu$ is $d_{1}$-optimal, then $\mu$ is straight.

Proof. Suppose for all $0<t<1$, Clearance $(\mu(t))>0$. Then it is an easy remark that $\mu$ must be straight.

Our goal is to construct a new motion $\mu^{\prime}$ with three properties: (1) Clearance $\left(\mu^{\prime}(t)\right)>$ 0 , (2) $\mu^{\prime}(t)=\mu(t)$ for $t=0,1$ and (3) $\mu, \mu^{\prime}$ have the same trace, $F \mu^{\prime}=F \mu$.

From (3), we have $d_{1}\left(\mu^{\prime}\right)=d_{1}(\mu)$ (= length of their traces). Since $\mu$ is optimal, and from (2), we conclude that $\mu^{\prime}$ must be optimal. From (1) and the easy remark, $\mu^{\prime}$ must be straight. Using (3) again, it follows that $\mu$ is also straight, proving our lemma.

Hence it remains to construct $\mu^{\prime}$. Towards this end, consider $\mu^{\prime \prime}=\rho \circ \mu$ (composition of $\rho$ with $\mu$ ). We see that $F \mu^{\prime \prime}=F \mu$. Furthermore, Lemma 9 tells us that $H(\mu(t))>0$ for all $0<t<1$. Thus Clearance $\left(\mu^{\prime \prime}(t)\right)=H(\mu(t))>0$. So, $\mu^{\prime \prime}$ has properties (1) and (3) needed for $\mu^{\prime}$. We can get (2) by concatenating a rotation at the beginning and at the end of $\mu^{\prime \prime}$. So what else is lacking? Unfortunately, $\mu^{\prime \prime}$ can have discontinuities: this happens at those $0<t_{0}<1$ where $\mu\left(t_{0}\right)$ is critical. By Sard's lemma [17], [18], we may assume that there only finitely many such discontinuities, which must be isolated. Now $\mu^{\prime}$ is obtained from $\mu^{\prime \prime}$ by "patching up" each of these discontinuities via a rotation at each discontinuity.

To see how this patchwork is achieved, observe that an isolated discontinuity at $t_{0}$ means that $\mu^{\prime \prime}\left(t_{0}^{-}\right) \neq \mu^{\prime \prime}\left(t_{0}^{+}\right)$. Moreover, $\mu^{\prime \prime}\left(t_{0}^{+}\right)$and $\mu^{\prime \prime}\left(t_{0}^{-}\right)$must be the two local maximas for the clearance function as we rotate $\mu\left(t_{0}\right)$ in the $\mathrm{CW}$ and CCW directions. We can connect $\mu^{\prime \prime}\left(t_{0}^{-}\right)$to $\mu^{\prime \prime}\left(t_{0}^{+}\right)$by a rotation motion that passes through $\mu\left(t_{0}\right)$. The minimum clearance achieved by this rotation motion is equal to Clearance $\left(\rho\left(\mu\left(t_{0}\right)\right)\right)=$ Clearance $\left(\mu\left(t_{0}\right)\right)>0$. In other words, by inserting such rotation motions into $\mu^{\prime \prime}$, we preserve the property that Clearance $\left(\mu^{\prime}(t)\right)>0$. This completes the description of $\mu^{\prime}$.

We next show two more preliminary results about constricted motions. If $p \in \mathbb{R}^{2}$ and $\varepsilon>0$, let $B_{\varepsilon}(p) \subseteq \mathbb{R}^{2}$ denote the open ball of radius $\varepsilon>0$ centered at $p \in \mathbb{R}^{2}$. For $Z \in F P$, define the reachable ball $B_{\varepsilon}(Z) \subseteq F P$ comprising those placements $Z^{\prime}$ that can be reached by a motion $\mu:[0,1] \rightarrow F P$ where the trace $F \mu$ is restricted to $B_{\varepsilon}(F[Z])$. Define the set of $\varepsilon$-accessible points from $Z$ to be $\left\{F\left[Z^{\prime}\right]: Z^{\prime} \in B_{\varepsilon}(Z)\right\}$, and denoted $F B_{\varepsilon}(Z)$. Clearly, $F B_{\varepsilon}(Z) \subseteq B_{\varepsilon}(F[Z])$. Also, $B_{\varepsilon}(F[Z]) \backslash F B_{\varepsilon}(Z)$ is the set of $\varepsilon$-inaccessible points from $Z$. 


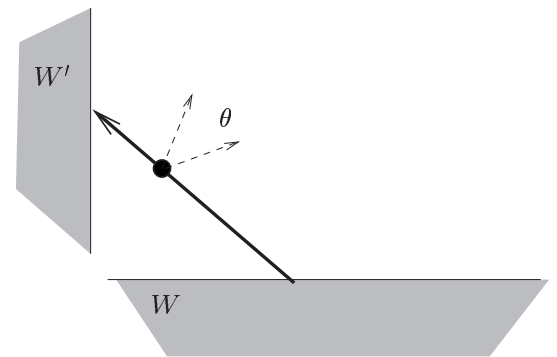

(a) Elliptic trace

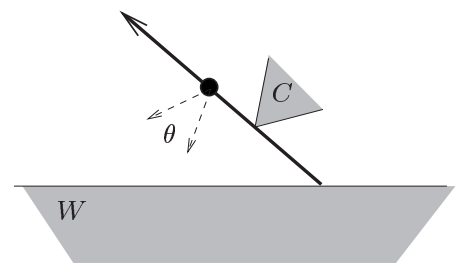

(c) Upper conchoid trace

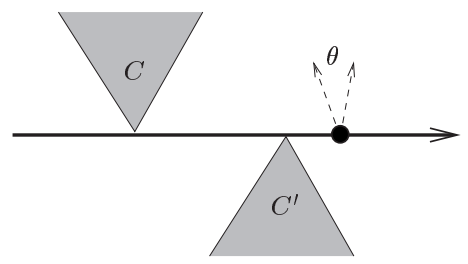

(e) Straight trace

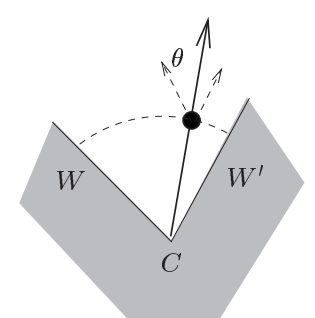

(b) Circular trace

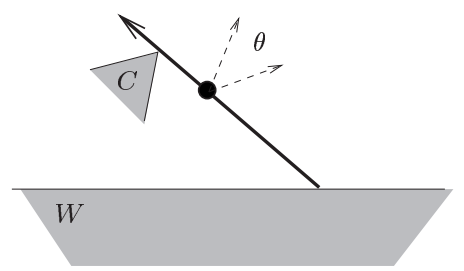

(d) Lower conchoid trace

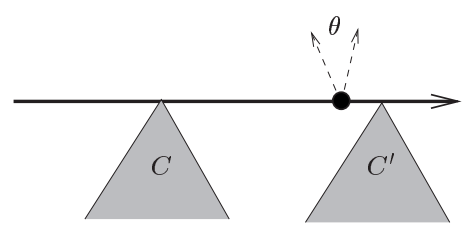

(f) Straight trace

Fig. 7. Local geometry of constricted motions.

Lemma 11. Let $\mu:[0,1] \rightarrow F P$ be a constricted edge. Then the trace $F \mu$ is the boundary of the locally accessible points. That is, for any $0<t<1$, there is an $\varepsilon>0$ such that

(i) The $\varepsilon$-accessible points $F B_{\varepsilon}(\mu(t))$ and the $\varepsilon$-inaccessible points $B_{\varepsilon}(F \mu(t)) \backslash F B_{\varepsilon}$ $(\mu(t))$ are connected sets.

(ii) The restriction of $F \mu$ to $B_{\varepsilon}(\mu(t))$ is a connected curve that separates $F B_{\varepsilon}(\mu(t))$ from $B_{\varepsilon}(F \mu(t)) \backslash F B_{\varepsilon}(\mu(t))$.

Proof. As illustrated in Fig. 7, the trace $F \mu$ is one of four types: (a) is an elliptic arc, (b) is a circular arc, (c) and (d) represent upper and lower conchoid arcs, while (e) and (f) are straight segments. To see that the trace $F \mu$ forms the boundary for the locally accessible points, we note that for any $0<t<1$, there is an open range $R=R(t)$ of angles at $F \mu(t)$ such that for any $\theta \in R$, there is a feasible motion starting from $\mu(t)$ with the trace moving in the direction $\theta$. Moreover, every motion starting from $\mu(t)$ in the opposite direction $-\theta$ is infeasible. 


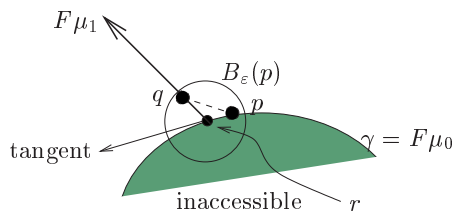

(a)

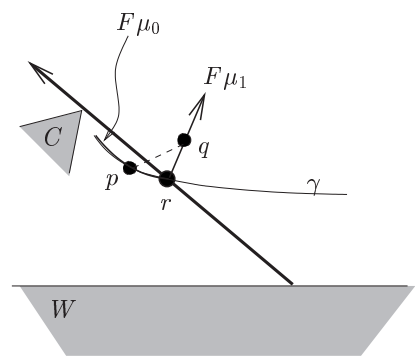

(b)

Fig. 8. Transition between constricted motion $\mu_{0}$ and unrestrained motion $\mu_{1}$ : (a) convex case, (b) nonconvex case.

Next we analyze how a restrained motion and a constricted motion can be joined together in an optimal motion. We first introduce the necessary notation. Let $\mu:[a, b] \rightarrow$ $F P$ and $\mu^{\prime}:\left[a^{\prime}, b^{\prime}\right] \rightarrow F P$ be two motions. We can concatenate them provided $b=a^{\prime}$ and $\mu(b)=\mu^{\prime}\left(a^{\prime}\right)$ and obtain a new motion denoted $\mu^{\prime \prime}:\left[a, b^{\prime}\right] \rightarrow F P$ where $\mu^{\prime \prime}(t)=$ $\mu(t)$ if $t \in[a, b]$ and $\mu^{\prime \prime}(t)=\mu^{\prime}(t)$ otherwise. We also write $\mu^{\prime \prime}=\mu$; $\mu^{\prime}$ for the concatenation of $\mu$ and $\mu^{\prime}$.

Lemma 12. Let $\mu=\mu_{0} ; \mu_{1}$ be $d_{1}$-optimal with $\mu_{0}$ constricted and $\mu_{1}$ unrestrained. Let $Z$ be the placement where $\mu_{0}$ joins $\mu_{1}$. Assume $F \mu_{0}$ and $F \mu_{1}$ are both non-constant functions and the set of $\varepsilon$-inaccessible points from $Z$ is convex. Then $F \mu_{0}$ is straight and connects to $F \mu_{1}$ tangentially at the point $F[Z]$.

Proof. Since $\mu_{1}$ is unrestrained, its trace $F \mu_{1}$ is straight. By Lemma $11, F \mu_{0}$ locally bounds the inaccessible points. Thus the tangent lines of $\mu_{1}$ (locally) lies in the accessible region. Let $F \mu_{0}$ meet $F \mu_{1}$ at the point $r$. See Fig. 8(a). If $F \mu_{1}$ is not a tangent to $F \mu_{0}$ at $r$, we can construct a shorter motion to obtain a contradiction. To do this, take a neighborhood $B_{\varepsilon}(r)$ as in Lemma 11 . Join a point $p$ on the $F \mu_{0}$ to a point $q$ on $F \mu_{1}$ by a line segment within this neighborhood. This segment is in the locally accessible region and $\mu$ can be modified to take a shortcut by tracing this segment.

The set of $\varepsilon$-inaccessible points from a non-vertex constricted $Z$ is generally convex for $\varepsilon$ sufficiently small (being bounded by straight segments or arcs of ellipses, circles or conchoids). Unfortunately, there is an exception: when the arc is the part of a conchoid that is closer to the directrix of the conchoid than to the pole. In this case the $\varepsilon$-accessible points need not be convex. This is illustrated in Fig. 8(b). We treat this case next.

Lemma 13. Let $\mu=\mu_{0} ; \mu_{1}$ be a motion with $\mu_{0}$ constricted and $\mu_{1}$ unrestrained. Let $Z$ be the placement where $\mu_{0}$ joins $\mu_{1}$. Assume $F \mu_{0}$ and $F \mu_{1}$ are both non-constant functions and for all $\varepsilon>0$, the set of $\varepsilon$-accessible points from $Z$ is non-convex. Then $\mu$ is not optimal. 
Proof. If $\mu$ is optimal, then $\mu_{1}$ is straight. Moreover, the trace of $\mu_{0}$ is part of a conchoid curve $\gamma$ (see Fig. 8(b)). We can choose a point $p=F \mu_{0}\left(t_{0}\right)$ and a point $q=F \mu_{1}\left(t_{1}\right)$ (for suitable $\left.t_{0}, t_{1}\right)$, both sufficiently close to $F[Z]$, such that there exists a straight motion from $\mu_{0}\left(t_{0}\right)$ to $\mu_{1}\left(t_{1}\right)$. This proves the non-optimality of $\mu$.

Proof of the Local Characterization (Theorem 5). Let $F \mu$ be locally non-straight at $t_{0}, 0<t_{0}<1$. If $\mu\left(t_{0}\right)$ is locally a vertex at $t_{0}$, then we satisfy the first condition in the theorem. In the rest of this proof we assume otherwise.

Claim. $\mu$ is locally restrained at $t_{0}$, i.e., if $I$ is any $F \mu$-neighborhood of $t_{0}$, there exists some $t \in I$ such that $\mu(t)$ is restrained.

By way of contradiction, assume the claim is false. Then Lemma 10 tells us that $\mu$ restricted to $I$ is straight. This contradicts our assumption that $\mu$ is not locally straight at $t_{0}$.

From this claim, we conclude that $\mu$ must be locally pivotal, constricted or reflecting at $t_{0}$. We consider each possibility in turn.

(i) Suppose $\mu$ is locally pivotal at $t_{0}$. That it is clear that $\mu\left(t_{0}\right)$ is in fact pivotal, i.e., there is a convex corner $C$ such that $F \mu\left(t_{0}\right)=C$. Let $I=\left[t_{1}, t_{2}\right]$ be the maximal interval containing $t_{0}$ such that $F \mu(t)=C$ for all $t \in I$. Since $t_{0}$ is not locally a vertex, it means that there exists an $\varepsilon>0$ such that $\mu \mid\left[t_{1}-\varepsilon, t_{1}\right]$ and $\mu \mid\left[t_{2}, t_{2}+\varepsilon\right]$ is unrestrained and thus straight. Moreover, the trace of these two straight motions must "bend" around $C$ because of optimality.

(ii) Suppose $\mu$ is locally constricted at $t_{0}$. Again, we conclude that $\mu\left(t_{0}\right)$ is constricted. So let $I=\left[t_{1}, t_{2}\right]$ be the maximal interval containing $t_{0}$ such that $F \mu(t)$ is constricted for all $t \in I$. We have two possibilities: (a) If $t_{0}=t_{1}$ or $t_{0}=t_{2}$, then $\mu\left(t_{0}\right)$ is the transition between a constricted motion $\mu \mid\left[t_{1}, t_{2}\right]$ and some other motion, say $\mu^{\prime}$. Since $\mu\left(t_{0}\right)$ is not locally a vertex, we conclude that $\mu^{\prime}$ cannot be restrained. By Lemmas 12 and 13, we conclude that $F \mu^{\prime}$ is straight, and meets the trace $F \mu_{i}$ tangentially at $\mu\left(t_{0}\right)$. (b) If $t_{1}<t_{0}<t_{2}$, then $\mu\left(t_{0}\right)$ is locally tracing a stopover curve.

(iii) Finally, assume $\mu\left(t_{0}\right)$ is locally reflecting. Let $I=\left[t_{1}, t_{2}\right]$ be the essential $F \mu$ neighborhood of $t_{0}$. Then there is some $t_{1} \in I$ where $\mu\left(t_{3}\right) \in M$, where $M$ is a mirror. Without loss of generality, let $A\left[\mu\left(t_{3}\right)\right]$ lie in some wall or corner. According to our analysis of mirrors in Appendix II, $\mu\left(t_{3}\right)$ satisfies some edge constraint of the form $\left[A^{+} @ s\right] \wedge\left[A^{-} @ s^{\prime}\right]$ where $s, s^{\prime}$ are two features. There are three possibilities (a) $s=s^{\prime}$ are the same wall, (b) $s$ is a corner and $s^{\prime}$ is an incident wall (or vice versa), (c) $s, s^{\prime}$ are the two walls incident on a common corner. For all sufficiently small $\varepsilon>0$, the points $F \mu\left(t_{1}-\varepsilon\right), F \mu\left(t_{3}\right), F \mu\left(t_{2}+\varepsilon\right)$ are not collinear. This is just our assumption that $\mu$ is not locally straight at $t_{0}$. Let $R \subseteq \mathbb{R}^{2}$ denote the zone of the mirror $M$ - this is the region $\bigcup\{A F[Z]: Z \in M\}$. Now, if $F \mu\left(t_{1}-\varepsilon\right)$ or $F \mu\left(t_{2}+\varepsilon\right)$ lies outside the zone $R$, then it is not hard to see that we can define a straightline motion from $\mu\left(t_{1}\right)$ to $\mu\left(t_{2}\right)$. This is a shortcut, contradicting the $d_{1}$-optimality of $\mu$. So both points lies in the zone. This means that $\mu\left(t_{1}-\varepsilon\right)$ satisfies $\left[A^{+} @ s\right]$ and $\mu\left(t_{2}+\varepsilon\right)$ satisfies $\left[A^{-} @ s^{\prime}\right]$ (the symmetric case is treated similarly). In this case the optimal motion from $\mu\left(t_{1}-\varepsilon\right)$ to $\mu\left(t_{2}+\varepsilon\right)$ must have a trace that reflects off the mirror curve of $M$ according to Snell's law, as claimed.

This completes the proof of Theorem 5 . 


\section{References}

1. L. Aleksandrov, M. Lanthier, A. Maheshwari, and J.-R. Sack. An $\varepsilon$-approximation scheme for weighted shortest paths. Proc. 6th SWAT, pp. 11-22. Lecture Notes in Computer Science 1432. Springer-Verlag, Berlin, 1998.

2. L. Aleksandrov, A. Maheshwari, and J.-R. Sack. An approximation scheme for geometric shortest path problems. Proc. 32nd STOC, pp. 286-295, 2000.

3. T. Asano, D. Kirkpatrick, and C. Yap. $d_{1}$-Optimal motion of a rod. Proc. 12 th ACM Symp. on Computational Geometry, pp. 252-263, 1996.

4. J. L. Bentley and A. C. Yao. An almost optimal algorithm for unbounded searching. Inform. Process. Lett., 5:82-87, 1976.

5. A. S. Besicovitch. On Kakeya's problem and a similar one. Math. Z., 27:312-320, 1928.

6. J. Canny and J. H. Reif. New lower bound techniques for robot motion planning problems. Proc. IEEE 28th Symp. on Foundations of Computer Science, pp. 49-60, 1987.

7. J. Choi, J. Sellen, and C. Yap. Approximate Euclidean shortest path in 3-space. Internat. J. Comput. Geom. Appl., 7(4):271-295, 1997. Also: Proc. 10th ACM Symp. on Computational Geometry, pp. 41-48, 1994.

8. K. L. Clarkson. Approximation algorithms for shortest path motion planning, Proc. 19th STOC, pp. 56-65, 1987.

9. T. H. Corman, C. E. Leiserson, R. L. Rivest, and C. Stein. Introduction to Algorithms, second edition. The MIT Press and McGraw-Hill, Cambridge, MA, and New York, 2001.

10. D. Halperin, L. Kavraki, and J.-C. Latombe. Robotics. In J. E. Goodman and J. O'Rourke, editors, Handbook of Discrete and Computational Geometry, chapter 41, pages 755-778. CRC Press, Boca Raton, FL, 1997.

11. J. Hershberger and L. J. Guibas. An $O\left(n^{2}\right)$ shortest path algorithm for non-rotating convex bodies. J. Algorithms, 9:18-46, 1988.

12. C. Icking, G. Rote, E. Welzl, and C. Yap. Shortest paths for line segments. Algorithmica, 10:182-200, 1993.

13. C. Ó’Dúnlaing, M. Sharir, and C. K. Yap. Retraction: a new approach to motion-planning. Proc. 15th ACM Symp. on Theory of Computing, pp. 207-220, 1983.

14. J. O'Rourke. Finding a shortest ladder path: a special case. IMA Preprint Series 353, Institute for Mathematics and its Applications, University of Minnesota, 1987.

15. C. H. Papadimitriou. An algorithm for shortest-path motion in three dimensions. Inform. Process. Lett., 20:259-263, 1985.

16. C. H. Papadimitriou and E. B. Silverberg. Optimal piecewise linear motion of an object among obstacles. Algorithmica, 2:523-539, 1987.

17. J. T. Schwartz and M. Sharir. On the piano movers' problem: I, the case of a two-dimensional rigid polygonal body moving amidst polygonal barriers. Comm. Pure Appl. Math., 36:345-398, 1983.

18. J. T. Schwartz, M. Sharir, and J. Hopcroft, editors. Planning, Geometry and Complexity of Robot Motion. Ablex Series in Artificial Intelligence. Ablex, Norwood, NJ, 1987.

19. J. Sellen, J. Choi, and C. Yap. Precision-sensitive Euclidean shortest path in 3-Space. SIAM J. Comput., 29(5):1577-1595, 2000. Also: Proc. 11th ACM Symp. on Computational Geometry, pp. 350-359, 1995.

20. M. Sharir. A note on the Papadimitriou-Silverberg algorithm for planning optimal piecewise-linear motion of a ladder. NYU Robotics Report 188, Courant Institute, New York University, 1989.

21. M. Sharir, C. Ó’Dúnlaing, and C. Yap. Generalized Voronoi diagrams for moving a ladder, I: topological analysis. Comm. Pure Appl. Math., XXXIX:423-483, 1986.

22. M. Sharir, C. Ó’Dúnlaing, and C. Yap. Generalized Voronoi diagrams for moving a ladder, II: efficient computation of the diagram. Algorithmica, 2:27-59, 1987.

23. Z. Sun and J. H. Reif. BUSHWHACK: an approximation algorithm for minimal paths through pseudoEuclidean space. Proc. 12th ISAAC, pp. 160-171. Lecture Notes in Computer Science 2223. SpringerVerlag, Berlin, 2001.

24. S. M. Ulam. Problems of Modern Mathematics. Science Editions, New York, 1964. Originally published as: A Collection of Mathematical Problems, Interscience, New York, 1960.

25. C. K. Yap. Algorithmic motion planning. In J. T. Schwartz and C. K. Yap, editors, Advances in Robotics, Vol. 1: Algorithmic and Geometric Issues, chapter 3. Erlbaum, Hillsdale, NJ, 1987. 